\title{
Formación de la pareja y fecundidad: una simulación con datos de la Comunidad de Madrid
}

\author{
Miguel Requena * \\ Departamento de Sociología II (Estructura Social) \\ Centro de Estructuras Sociales Comparadas \\ Universidad Nacional de Educación a Distancia \\ mrequena@poli.uned.es
}

Every obstacle in the way of marriage must undoubtedly be considered as a species of unhappiness. But as from the laws of our nature some check to population must exist, it is better that it should be checked from a foresight of the difficulties attending a family and the fear of dependent poverty than that it should be encouraged, only to be repressed afterwards by want and sickness.

Thomas Robert Malthus, An Essay on the Principle of Population (1798)

\section{INTRODUCCIÓN}

En los últimos tiempos se han multiplicado los diagnósticos que nos advierten que nuestro país ha ingresado, al igual que otras sociedades europeas, en un régimen de muy baja o bajísima fecundidad. En efecto, durante los últimos veinticinco años los índices coyunturales de fecundidad han venido experimentado en España una acusadísima disminución que nos ha llevado a ocupar, junto con Italia y algunos otros países de Europa oriental, los últimos lugares del mundo. Como es

* El autor quiere expresar su agradecimiento a los participantes en el seminario del CESC por sus amables comentarios y atinadas sugerencias. De particular valor han sido las críticas de Fabrizio Bernardi, Fermín Bouza, M.a Antonia Carbonero, Elisa Chulia, Marta Fraile, Luis Garrido, M. ${ }^{a}$ Victoria Gomez, Teresa Jurado, Emilio Luque, Miguel Ángel Malo, Jaime Riviere y Carmen TORRALBo. Los posibles errores son exclusiva responsabilidad del autor. 
bien sabido, esa reciente caída se inscribe en una consolidada tendencia secular que ha ido rebajando de forma sostenida la intensidad del comportamiento reproductivo de las mujeres españolas (Fernández, 1986; Requena, 1997; Pérez Díaz, 2002) y que está asociada a diferentes procesos de cambio demográfico, social, económico y cultural cuyos efectos se han combinado para desincentivar radicalmente la producción de hijos. Todos esos procesos, y en particular los que han alterado la posición social de las mujeres y las estructuras familiares, vienen operando con mayor o menor pujanza en todas las sociedades avanzadas (Garrido, 1996). Pero lo notable en países como el nuestro es que en los últimos años el fenómeno de la escasa fecundidad ha alcanzado tal magnitud que, de mantener a lo largo de su vida fértil la intensidad genésica que exhiben hoy, las mujeres españolas no tendrían por término medio más de 1,2 hijos a la conclusión de su calendario reproductivo.

A esa nueva situación en la que los indicadores coyunturales no registran más de 1,3 hijos por mujer, un nivel muy inferior al de reemplazo generacional, es precisamente a la que se califica de régimen de muy baja fecundidad (Köhler, Billari y Ortega, 2001). Buen ejemplo de tal régimen es la Comunidad de Madrid, donde el índice sintético de fecundidad viene cayendo de manera continuada desde 1976. Las mujeres residentes en esta comunidad, que constituyen el soporte empírico del argumento que desarrollaré en este trabajo, presentaban en 1998 una fecundidad coyuntural de 1,16 hijos. Y, desde que en 1989 se alcanzara por primera vez el promedio de 1,3 hijos por mujer, la Comunidad de Madrid se ha mantenido durante toda la década de 1990 en un régimen de muy baja fecundidad. Conocidos, como son, todos esos datos, casi resulta ocioso insistir en que la contemporánea caída de los niveles reproductivos de nuestra sociedad ha despertado alarma en ciertos sectores de opinión, además de preocupación entre los administradores públicos e interés entre los expertos.

En el presente trabajo me propongo establecer que la reciente caída de la fecundidad de las mujeres en las sociedades con comportamientos demográficos típicamente sudeuropeos se debe en muy buena medida, y cualquiera que sea el impacto final que acabe teniendo en la descendencia final de las generaciones, a los problemas relacionados con la formación de matrimonios y parejas; a su vez, las dificultades que rodean el establecimiento de matrimonios y parejas y, por ende, de nuevas unidades familiares, mantienen una estrecha relación con los obstáculos que se han encontrado los jovenes en su esfuerzo por incorporarse al mundo adulto. La interpretación que presento de la caída de los niveles reproductivos en las sociedades sudeuropeas se basa en un ejercicio empírico de descomposición del índice sintético de fecundidad que, realizado por dos métodos diferentes, ilustra de manera muy convincente el viejo argumento demográfico de la conexión causal entre nupcialidad y fecundidad. La evidencia que aporto en este trabajo permite comprobar con un alto grado de certidumbre dicha conexión a partir de los datos de la comunidad madrileña, al menos durante los años ochenta y noventa del pasado siglo.

Para ese periodo las dificultades relativas a la emancipación de los jóvenes en España y en Madrid -la creciente dependencia familiar de sus padres y el retraso en el calendario de su independencia doméstica- están bien establecidas y han sido objeto de análisis muy detallados (véanse, por ejemplo, Garrido y Requena, 1987; Garrido y Requena, 1995; Garrido y Requena, 1996; Fernández, 1997; Martínez Gra- 
nado y Ruiz Castillo, 1998; Requena, 1999; Jurado, 2001; Requena, 2001; y Requena, 2002). Paralelamente, varios trabajos (Garrido, 1993; Garrido y Requena, 1996; Gil y Cabré, 1997; y Puyol, 1999) han postulado ya explícitamente la conexión causal entre las crecientes dificultades para la independencia doméstica de los jóvenes, la dificultades para estabilizar las parejas y la intensa caída de la fecundidad. Un reciente estudio sobre el régimen de bajísima fecundidad de las sociedades avanzadas (Köhler, Billari y Ortega, 2001) señala cómo la gran incertidumbre económica en que se viven los comienzos de la vida adulta se ha convertido, sobre todo en los paises del sur de Europa, en un factor determinante del retraso en el calendario reproductivo y del correlativo descenso en los indicadores coyunturales de fecundidad.

Como he señalado más arriba, la conexión causal entre nupcialidad y fecundidad no es, desde luego, un argumento demográfico nuevo. Es evidente que allí donde una gran mayoría de los hijos nace en el seno de la institución del matrimonio, la producción de niños depende crucialmente de la cantidad de individuos comprometidos con la vida conyugal; en tales condiciones, las tasas de fecundidad son una función de la proporción de mujeres en edad fecunda que se encuentran casadas. Hay que resaltar, a este respecto, que el nivel de fecundidad de una población es muy sensible a la proporción de sus mujeres casadas y que este factor presenta, además, una gran variabilidad entre diferentes poblaciones y a lo largo del tiempo, dos características que lo convierten en pieza clave en la modelización teórica (Bongaarts, 1982) de los llamados determinantes próximos o variables intermedias de la fecundidad ${ }^{1}$. En lógica consecuencia, muchos estudios tanto de historia de la población -véase, por ejemplo, Wrigley (1992)-como de diferentes procesos contemporáneos de disminución de la fecundidad - véanse, por ejemplo, los trabajos de Wunsch (1982) para diversos países europeos y de Limanonda (1992) para el caso tailandés-, han recurrido a explotar las implicaciones analíticas y empíricas de dicha conexión.

No es ésta, tampoco, la primera vez que se trabaja empíricamente sobre la relación entre nupcialidad y fecundidad en la interpretación de los cambios en los niveles de la segunda en España. Así, por ejemplo, varios estudios de demografía histórica han puesto de manifiesto las complejas relaciones entre nupcialidad, fecundidad general y fecundidad matrimonial que se han dado en nuestro país durante el proceso de su transición demográfica, complejidad que incluye una notable diversidad de pautas regionales (Reher, 1996). Cabe destacar, en esta línea, el examen del sistema catalán de reproducción realizado con amplia perspectiva histórica por Cabré (1999), quien aborda el análisis de la relación entre nupcialidad y fecundidad a partir de la descomposición de la fecundidad general propuesta por Coale -con una interesante aplicación a los datos generacionalespara plantear que, cuando menos durante el siglo veinte, el comportamiento reproductivo de diferentes cohortes de mujeres catalanas se explica a partir precisamente de los cambios habidos en la nupcialidad. Las conclusiones de la autora son muy claras a este respecto: mientras la declinante fecundidad de las gene-

1 Según el modelo de Bongaarts, la proporción de mujeres casadas y el uso de anticonceptivos son de hecho las dos variables intermedias o determinantes próximos de la fecundidad que tienen más importancia. 
raciones catalanas nacidas en el siglo XIX se explica sobre todo por la disminución de la fecundidad matrimonial, «las variaciones de la fecundidad de las generaciones del siglo XX se explican más por los cambios de la nupcialidad que por el comportamiento de los matrimonios» (Cabré, 1999, 117).

Entre los estudios referidos al periodo más reciente de intensa caída de nuestros niveles reproductivos, se pueden citar algunos ejemplos que abundan en la relación entre nupcialidad y fecundidad. En un análisis de comienzos de los años ochenta se partía de la hipótesis de que la «caída de la fecundidad general estaría a su vez ocasionada por la caída de la nupcialidad, al menos en términos transversales o coyunturales» (Agüero y Olano, 1982, 33). Un estudio posterior defendía que el «excepcionalmente bajo nivel de fecundidad registrado en España es parcialmente atribuible a las cambiantes pautas de formación de la familia entre las generaciones más jóvenes, en particular a la posposición del matrimonio y al aplazamiento de los primeros nacimientos» (Castro, 1992, 219); el detallado análisis sociodemográfico del retraso de la maternidad realizado por esta autora concluía que pese a las considerables diferencias respecto al momento en que se produce la transición al primer hijo, no se encontraba una gran variación en el salto a la maternidad una vez que se controlaba la duración del matrimonio, porque las madres retrasadas se terminaban poniendo al día. En otro estudio específicamente referido a la Comunidad de Madrid también se reconocía que la nupcialidad era «una variable importante en la determinación de los niveles de fecundidad» (Fernández, 1993, 63). En una evaluación de conjunto del crecimiento natural de la población española y sus determinantes (Gil y Cabré, 1997) se plantea explícitamente que la nupcialidad es un factor clave en la evolución de la fecundidad. Todos estos trabajos referidos a la intensa caída de la fecundidad acaecida en nuestro país en los últimos veinticinco años, aunque interesantes por muchos conceptos, se han limitado a señalar la convergencia espacial y temporal de los indicadores agregados de ambos fenómenos; sin embargo, no ofrecen información empírica detallada, ni medidas más o menos precisas, ni tampoco pruebas definitivamente concluyentes del efecto de la nupcialidad en la caída de la fecundidad. Mención aparte merece, sin embargo, el reciente trabajo de Delgado (2000), en el que se analiza con todo detalle el impacto de la nupcialidad en la reducción del promedio de hijos por mujer en España y, en especial, durante el periodo 19911996. Por mi parte, y en la línea de este último trabajo, me propongo ampliar el argumento de la nupcialidad al emparejamiento y fundamentar empíricamente, hasta donde ha sido posible, la conexión entre ambos fenómenos -emparejamiento y fecundidad- con datos que proceden de la Comunidad de Madrid.

Si los resultados que expongo en este trabajo no son incorrectos, se puede dar por documentada empíricamente, y en consecuencia por bien establecida en el ámbito poblacional elegido, una estrecha conexión causal entre emparejamiento y fecundidad, en el sentido de que la posposición del emparejamiento y la creciente soltería de los y las jóvenes adultos han rebajado drásticamente los niveles de la fecundidad de las madrileñas. Con ello se recupera, como queda dicho, un argumento clásico en el análisis demográfico de la fecundidad como es el de sus determinantes próximos, es decir, las variables intermedias a través de las cuales las condiciones sociales, económicas y culturales terminan por regular la 
intensidad de las conductas reproductivas en una determinada sociedad. Es bien cierto que en estos últimos años el interés por determinar la influencia del emparejamiento en la fecundidad ha disminuido mucho, y ello no es extraño si se tiene en cuenta la progresiva desinstitucionalización del matrimonio, la creciente difusión de la cohabitación entre los jóvenes de las sociedades avanzadas y la incidencia cada vez mayor de los nacimientos fuera del matrimonio (Muñoz, 1995; Valero y Lence, 1995). Todos esos fenómenos se han ido propagando, con mayor o menor fuerza, en los países del centro y del norte de Europa. Sin embargo, en las sociedades meridionales europeas que han ingresado en el actual régimen de muy baja fecundidad, el proceso de formación de la pareja parece haber recuperado en estos últimos años una de las funciones seculares que venía cumpliendo en el área europea occidental: la de regular preventivamente la capacidad reproductiva de sus poblaciones.

\section{TENDENCIAS}

En opinión de reputados demógrafos históricos, el matrimonio ha sido durante mucho tiempo entre las poblaciones de Europa occidental el verdadero gozne sobre el que ha girado todo el sistema demográfico. De acuerdo con Hajnal (1965), la pauta europea (occidental) de matrimonio consistía en una combinación de casamientos tardíos y celibato extendido y permanente que permitía a las poblaciones que la practicaban contar con un dispositivo muy depurado de control de la fecundidad ${ }^{2}$. La existencia del patrón matrimonial europeo occidental está documentada, al menos, desde el siglo XVIII y su vigencia se habría prolongado hasta la Segunda Guerra Mundial. Así las cosas, una de las formas de entender la transición demográfica en el área europea es como si hubiera consistido en el proceso de desaparición de esa pauta nupcial. Con el recurso generalizado a la anticoncepcion, el matrimonio se rejuvenece y las dimensiones de la soltería se reducen drásticamente. En otros términos, el patrón nupcial típico de muchas sociedades del occidente europeo fue desapareciendo en la medida en que el matrimonio tardío y el celibato permanente iban perdiendo su tradicional función de controlar el tamaño de las familias ${ }^{3}$.

Por supuesto, las variedades territoriales -nacionales, regionales y aun locales- del cambio demográfico son tan numerosas, y su cronología tan distinta, que es difícil perfilar una interpretación unívoca del proceso de transición de la nup-

${ }^{2}$ Bajo los supuestos del modelo matrimonial europeo, el principal factor que influía en la fecundidad era la incidencia de la nupcialidad (VAN DE WALLE, 1978); el refinamiento del mecanismo matrimonial se encontraba, sobre todo, en la alta y rápida sensibilidad de la nupcialidad a las variaciones de la coyuntura economica y a las fluctuaciones de la mortalidad (WrigLeY: 1992).

${ }^{3}$ Como señaló un especialista: «... el descenso de la fecundidad que ha sido casi universal en Europa fue un cambio desde (a) una fecundidad moderada, que se mantuvo alejada de los niveles muy altos mediante el matrimonio tardío y el celibato permanente, y mediante la limitación específicamente no relacionada con la paridez de la fecundidad marital, a (b) una baja fecundidad producida fundamentalmente por la práctica específicamente relacionada con la paridez de la contracepción o el aborto» (COALE: 1986, 10). 
cialidad y la fecundidad en el área europea. En España, por ejemplo, la heterogeneidad en lo que se refiere a los comportamientos nupciales era ya muy considerable antes incluso de iniciarse la transición demográfica (Rowland, 1988), lo que sin duda ha debido generar una diversidad no menos notable de pautas de nupcialidad y natalidad durante la fase de transformación. En lo que aquí interesa más, parece que en muchas zonas de nuestro país hay que esperar a después de 1920 o 1930 para observar síntomas elocuentes del debilitamiento de la función tradicional del matrimonio en la regulación de la reproducción (Reher, 1996, 242). Por lo que sabemos de las características demográficas de los madrileños durante su historia más reciente, sus comportamientos nupciales se han ajustado a lo largo de gran parte del siglo XX a lo que se puede esperar del modelo europeo: es decir, condiciones sumamente restrictivas de acceso al matrimonio al comienzo del siglo, que se fueron relajando a medida que se progresaba en la modernización social y económica y se iba recorriendo el camino de la transición demográfica.

Aun a riesgo de simplificar en exceso, se puede tratar de hacer un sucinto resumen de la reciente historia demográfica de Madrid en la última centuria. En comparación con otras zonas españolas, los residentes en la antigua provincia (hoy Comunidad) de Madrid se han distinguido durante el siglo XX tanto por un calendario nupcial muy tardío, como por unas tasas de soltería sumamente altas, lo que ha llevado a un estudioso de nuestra historia demográfica a calificar la estructura matrimonial de Madrid como una de las más limitativas de España durante la primera mitad del siglo $\mathrm{XX}^{4}$ (Miret, 2002, 275). Sin embargo, es claro que, en términos de los indicadores transversales, durante la segunda mitad del siglo XX el modelo se va debilitando en Madrid, al menos en el sentido de que se casa mucha más gente (las tasas coyunturales de nupcialidad masculinas y femeninas van aumentando después de la Guerra Civil hasta alcanzar un máximo en torno a mediados de los años setenta) y de que se contrae matrimonio a una edad cada vez más temprana (disminuyendo así la edad promedio al primer matrimonio tanto de mujeres como de hombres hasta finales de los años setenta). Mediados los años setenta, las tasas coyunturales de nupcialidad registraban máximos históricos y el calendario matrimonial mostraba una insólita precocidad. El modelo europeo de matrimonio parecía cosa del pasado.

No obstante, desde mediados de los años setenta los datos transversales de la nupcialidad y la fecundidad apuntan a una nueva inversión del ciclo demográfico en Madrid, contraria por cierto a la supuesta tendencia a desaparecer del modelo europeo. Por lo que se refiere, en primer lugar, a la nupcialidad, bastará con seleccionar unos pocos indicadores que reflejan ese cambio de ciclo. Así, por ejemplo, si en 1975 sesenta y tres de cada cien mujeres madrileñas de entre 15 y 49 años estaban casadas, en 1996 sólo se encontraban en ese mismo estado civil cuarenta y ocho de cada cien. Esta fuerte reducción de la proporción de mujeres madrileñas casadas, del orden de un $30 \%$ en términos relativos, se corresponde con: $a$ ) una caída intensa de los índices de primonupcialidad, que disminuyen fuertemente desde 1975 a 1980 , ralentizan su caída en los primeros ochenta y se recuperan levemente en la segunda

${ }^{4}$ Los intensos flujos migratorios de solteros y solteras hasta la ciudad de Madrid constituyen uno de los factores que pueden haber pesado más decisivamente sobre su estructura nupcial. 
mitad de los ochenta, siempre sin remontar los niveles alcanzados a comienzos de los ochenta; y $b$ ) una significativa elevación de la edad de entrada al matrimonio de las mujeres y los hombres madrileños, que en el caso de las primeras se mueve desde los 24,4 años de 1975 hasta los 26,76 de 1991 (Domingo, 1997).

GRÁFICO 1

Índice sintético de fecundidad y la tasa bruta de natalidad en Madrid

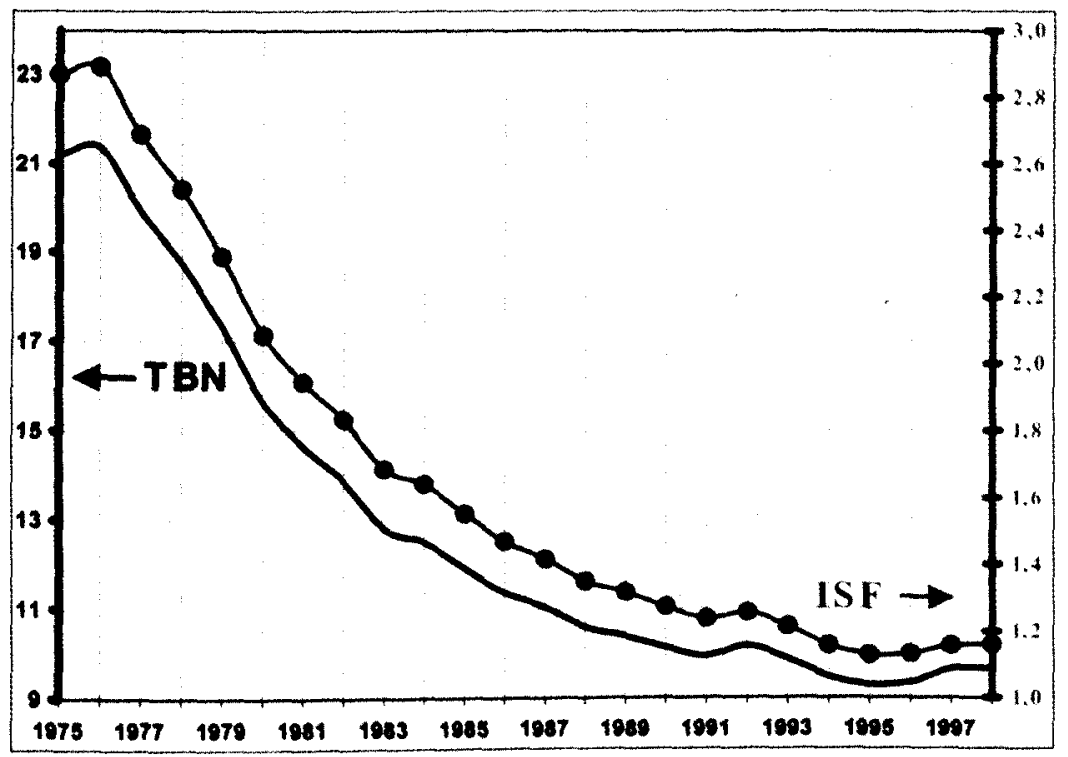

Fuente: Comunidad de Madrid.

En segundo lugar, tanto las medidas coyunturales de intensidad de la fecundidad como las de calendario registran el radical cambio de pautas reproductivas que han vivido las mujeres madrileñas durante estos años. En 1975 nacieron en Madrid 90801 niños; veinte años más tarde esa cifra se había reducido hasta los 47483 nacimientos de 1996; en el mismo periodo las posibles madres de esos hijos (las mujeres de entre 15 y 49 años) habían aumentado desde 1,087 hasta 1,424 millones. $\mathrm{El}$ índice sintético de fecundidad (ISF) ha experimentado desde 1975 hasta 1998 un descenso sostenido, desde los 2,87 hijos por mujer hasta 1,16 , una evolución muy paralela a la de la tasa bruta de natalidad, que ha disminuido desde los 21 nacimientos por mil habitantes de 1975 hasta los sólo 9,6 por mil de 1998 (gráfico 1). La caída del ISF es muy pronunciada al comienzo del periodo considerado -durante la segunda mitad de los setenta y primeros ochenta-, para atenuarse después y estabilizarse en la segunda mitad de los años noventa en unos niveles ya muy bajos, desde los que se atisba incluso algún síntoma de recuperación los últimos años. En todo caso, el ISF se sitúa desde 1980 por debajo del nivel de reemplazo generacional y hacia 1990 desciende por debajo del umbral que define a los regímenes contemporáneos de muy baja fecundidad (fecundidad coyuntural de 1,3 hijos por mujer). 
GRÁFICO 2

Tasas de fecundidad por tramos de edad en la Comunidad de Madrid

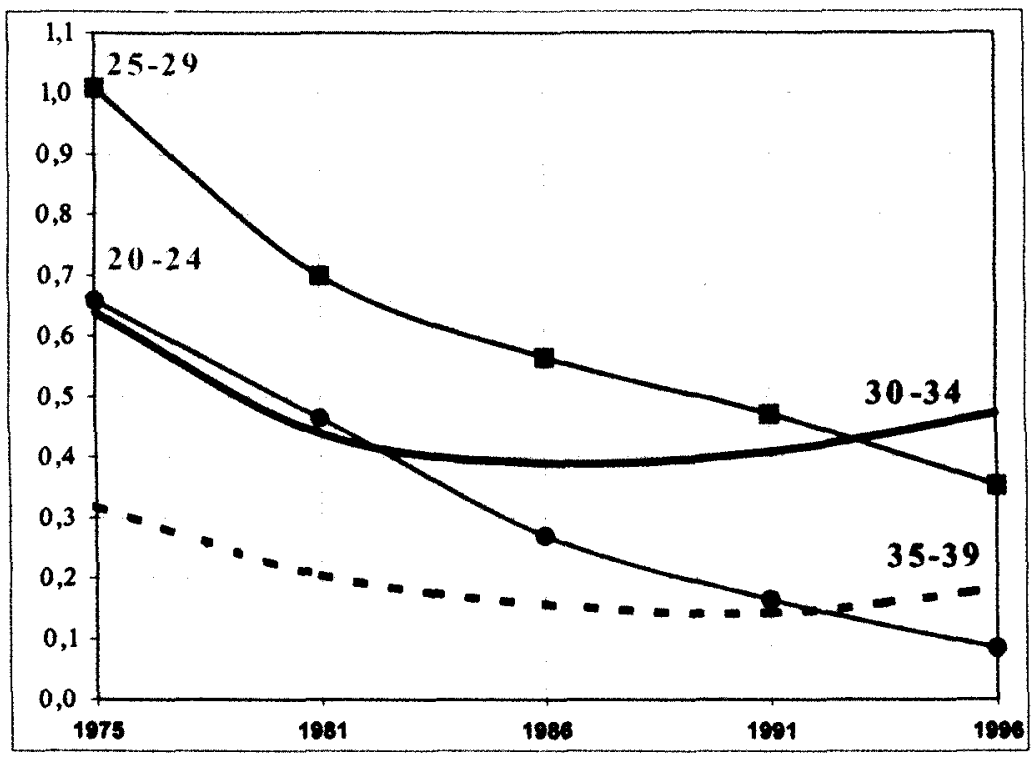

Fuente: Censos y Padrones de Población y Movimiento Natural de la Población.

En cuanto al calendario de la reproducción, varios indicadores reflejan también las importantes transformaciones de las pautas de distribución de la fecundidad en el ciclo vital de las mujeres madrileñas. Por ejemplo, la edad media a la maternidad del primer hijo se ha elevado desde los 25,08 años en 1975 hasta los 29,91 en 1998, lo que supondría (si el dato transversal se proyectara tal cual a la vida reproductiva de las generaciones reales) un retraso de casi cinco años $y$, por consiguiente, una pérdida promedio de aproximadamente el $14 \%$ del tiempo total de duración del ciclo reproductivo femenino. Tiene interés señalar asimismo la evolución de las tasas específicas por edad de fecundidad (gráfico 2) Mientras al comienzo del periodo, el segmento de edad más fecundo era el comprendido entre los 25 y los 29 años, a finales de los años noventa son las edades comprendidas entre los 30 y los 34 años las que presentan mayores tasas de fecundidad. Las mujeres de entre 20-24 y 25 29 años han experimentado un descenso constante y muy acusado de sus tasas de fecundidad, habiendo pasado el tramo 20-24 años a ser el menos fecundo al final del periodo. La fecundidad de las madrileñas de 25-29 años, la más alta en los años setenta, cayó por debajo de las de 30-34 años. Finalmente, no se puede obviar el hecho de que tanto las mujeres madrileñas de entre 30-34 años, como las de entre 35-39, tras reducir su fecundidad entre 1975 y 1981 , prácticamente la estabilizan durante las décadas de los años ochenta e incluso la recuperan en los noventa. 
Gráfico 3

Evolución del número de nacimientos por estado civil de la madre en la Comunidad de Madrid y proporción de nacimientos extramatrimoniales

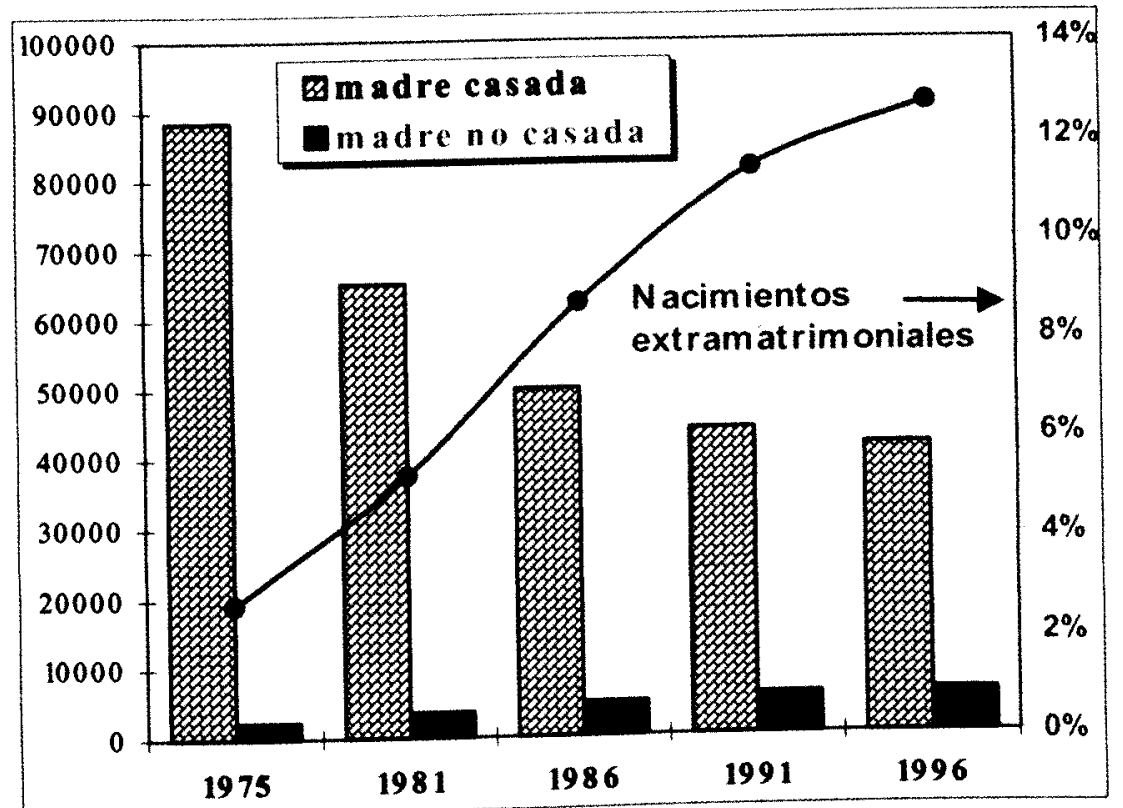

Fuente: Movimiento Natural de la Población.

Por último, es preciso mencionar brevemente la creciente incidencia de la fecundidad extramatrimonial en la Comunidad de Madrid, un fenomeno que se ha extendido a la par que la cohabitación y que resulta fundamental tener en cuenta cuando se pretende evaluar el impacto de los niveles de celibato en la fecundidad. El hecho es que desde 1975 en adelante la proporción de nacimientos que se producen fuera del matrimonio no ha dejado de crecer en Madrid. Las cifras son, en este sentido, terminantemente claras. En 1975, sólo tres de cada cien niños nacidos en la entonces provincia de Madrid eran hijos de madres no casadas. En 1996, esa proporción se había elevado hasta el 12\% (gráfico 3). Dos años más tarde, en 1998, los nacimientos extramatrimoniales constituían nada menos que un $17 \%$ del total de la comunidad madrileña.

En suma, los madrileños parecen haber desandado en los últimos veinticinco años parte del camino recorrido durante la transición demográfica, al menos en lo que se refiere a sus pautas y modelos matrimoniales. La evolución durante esos años de los indicadores de coyuntura demográfica de la Comunidad de Madrid apunta a una profunda depresión de la nupcialidad, con el resultado de que se ha vuelto a una situación de soltería creciente y de calendario matrimonial o de emparejamiento muy retrasado. La depresión de la nupcialidad ha tenido su correlato en una caída muy pronunciada y casi ininterrumpida de la fecundidad coyuntural de las mujeres madrileñas. ¿En qué medida están relacionados ambos 
fenómenos? ¿Hasta qué punto es responsable esa creciente soltería del desplome de la fecundidad de las madrileñas?

\section{MÉTODOS}

La idea básica para evaluar el impacto del creciente celibato en la caída de los niveles reproductivos registrados entre las mujeres madrileñas consiste en descomponer la fecundidad coyuntural observada a lo largo del tiempo en dos de sus componentes fundamentales: intensidad de las conductas de emparejamiento y fecundidad de las parejas. La fecundidad observada se evalúa con el índice sintético de fecundidad (o tasa de fecundidad total, TFR, en la nomenclatura anglosajona), una medida simple y de muy fácil interpretación que, sin embargo, presenta muchas limitaciones debido a que no refleja la experiencia vivida por ninguna generación real de mujeres y a que amalgama de un modo muy inconveniente los componentes de intensidad y calendario de la fecundidad ${ }^{5}$. Pese a todos los problemas que rodean al índice, pocos discuten la necesidad de recurrir a este tipo de medidas coyunturales o de periodo ${ }^{6}$. Por lo demás, en el marco de este ensayo usar el índice sintético de fecundidad parece lo más oportuno en la medida en que cuando se insiste en la caída reciente de la fecundidad se habla, sobre todo, de la evolución de ese índice.

La literatura especializada ofrece, como es sabido, diferentes métodos para descomponer los índices transversales de fecundidad ${ }^{7}$, pero en el contexto del presente argumento bastará con recurrir a un par de formas de descomposición que permiten aislar dos de los componentes fundamentales de estos índices: de un lado, la intensidad del emparejamiento (es decir, la proporción de mujeres en edad fértil que se encuentran casadas o emparejadas); y de otro, la fecundidad de los matrimonios o parejas. Esos dos componentes no agotan, desde luego, los factores relevantes que pesan sobre la fecundidad, pero separándolos se hace posible evaluar su importancia relativa en los resultados agregados del proceso reproductivo y, así, determinar de forma rigurosa la influencia de la soltería en la fecundidad. Además, una vez que se ha precisado el peso de esos dos componentes, no resulta difícil simular la posible evolución que habría experimentado la fecundidad agregada de las mujeres madrileñas si durante los últimos años hubiera cambiado solamente uno de ellos, por ejemplo, si hubiesen alterado sus pautas de fecundidad mientras mantenían constante su intensidad de emparejamiento.

5 Una propuesta para ajustar el índice de manera que se eliminen los efectos del calendario sobre el comportamiento de la fecundidad se encuentra en BonGAARTS y FEENEY (1998); véase también la crítica de VAN IMHOFF y KeILMAN (2000).

6 Para una defensa de las medidas de periodo, que no elude las deficiencias del ISF, y una crítica de las de cohorte, véase Ni BHrolcháin (1992).

7 Dos métodos que aquí no utilizaré son, por ejemplo, los propuestos por GraBILL, KISER y WhELPTON (1958) y el ya clásico en los estudios de demografía histórica de COALE (1969). Wunsch (1982) presenta una descomposición de la tasa bruta de natalidad basada en los trabajos de BerENT y Festy. Una aplicación de la descomposición de Coale a la fecundidad de Madrid se encuentra en Domingo (1997). 
Al objeto de realizar la descomposición, se seguirán dos métodos diferentes que, no obstante, conducen a conclusiones muy similares. El primero de ellos se basa directamente en la modelización teorica propuesta por Bongaarts (1978 y 1982) de los determinantes próximos de la fecundidad y en el desarrollo de su aparato metodológico. El segundo lleva a cabo la descomposición del ISF a partir de las tasas de fecundidad específicas por edad de las mujeres casadas y no casadas; a renglón seguido, y mediante un simple ejercicio de estandarización, se estima la fecundidad que se podría esperar que se hubiera producido entre las mujeres madrileñas si éstas hubieran mantenido las mismas pautas de emparejamiento que se daban en el año 1975.

El marco teórico de Bongaarts supone que una serie de variables intermedias o determinantes próximos -el celibato, el uso de métodos anticonceptivos, los abortos inducidos, la infecundidad posparto y la esterilidad patológica- actúan como factores inhibidores de la fecundidad. Que estos factores se consideren inhibidores se debe al hecho de que la fecundidad nunca se observa en su máximo nivel en las poblaciones reales; lo que se observa en la realidad es la tasa de fecundidad total (la TFR, versión anglosajona del denominado ISF en francés o castellano ${ }^{8}$ ), que es el nivel de fecundidad que consigue una determinada población una vez que los factores inhibidores han trasladado su efecto a la conducta reproductiva de las mujeres. El propio Bongaarts estimó que la fertilidad máxima, la que se produciría en ausencia de factores inhibidores, oscila en las poblaciones humanas entre los 13 y los 17 hijos por mujer, situándose la media en 15,3 hijos por mujer.

En lo que se refiere a celibato y matrimonio, la propuesta de Bongaarts consiste en definir un nivel puramente teórico de fecundidad matrimonial total (TMFR) -aquella fecundidad que se alcanzaría sin el efecto inhibidor del celibato sobre la tasa de fecundidad total-y compararla con la fecundidad realmente observada (TFR). El índice de matrimonio, $C_{m}$, es la razón entre la tasa total de fecundidad y la tasa de fecundidad matrimonial total $\left(C_{m}=T F R / T M F R\right)$ y expresa el grado en el que la soltería rebaja la posible fecundidad de los matrimonios hasta el nivel de la fecundidad observada: cuanto menor el índice de matrimonio, mayor la incidencia de la soltería en la fecundidad y mayor el descenso de la fecundidad observada respecto de la fecundidad matrimonial $\left(T F R=C_{m} x\right.$ $T M F R$ ). De esas relaciones se sigue que la razón entre la tasa de fecundidad total y el índice de matrimonio es igual a la tasa de fecundidad matrimonial total $\left(T F R / C_{m}=T M F R\right)$, la fecundidad que se observaría si las pautas de emparejamiento matrimonial no impusieran ninguna limitación a la fecundidad realmente observada.

Puesto que la tasa de fecundidad matrimonial total (TMFR) no se puede observar directamente, Bongaarts la estima como una razón entre las tasas de fecundidad y la proporción de mujeres casadas $(T M F R=\Sigma[f(x) / m(x)]=\Sigma g(x))$, donde $f(x)$ son las tasas de fecundidad específicas por edad y $m(x)$ las proporciones de mujeres casadas por edad). Para las edades fértiles, cada tasa específica de

8 Por razones de conveniencia, en el marco del análisis de Bongaarts utilizaré la denominación TFR. 
fecundidad se ve multiplicada por la probabilidad inversa de estar casada. Por lo tanto, el índice de matrimonio se calcula como sigue:

$$
C_{m}=\frac{T F R}{T M F R}=\frac{\sum_{x=15}^{x=49} f(x)}{\sum_{x=15}^{x=49} f(x) / m(x)}=\frac{\sum_{x=15}^{x=49} m(x) g(x)}{\sum_{x=15}^{x=49} g(x)}
$$

El índice, que oscila entre 0 (ninguna mujer se ha casado) y 1 (todas las mujeres se encuentran casadas durante todo su periodo reproductivo), viene a ser una media de la proporción de mujeres casadas ponderada por las tasas de fecundidad matrimonial que presentan a las diferentes edades de su vida fértil.

El método de Bongaarts se ha utilizado abundantemente para descomponer la contribución de los determinantes próximos a la fecundidad total, para analizar la aportación de las variables intermedias al cambio en el tiempo de la fecundidad total y también para comparar los resultados agregados de la fecundidad en distintos países y regiones a partir de las diferencias en los determinantes próximos. Sin embargo, uno de los inconvenientes que presenta esta descomposición, explícitamente reconocido por su autor, es que no tiene en cuenta los nacimientos que se producen fuera del matrimonio ${ }^{9}$, un fenómeno cada día más extendido en las sociedades avanzadas y del que también ha sido testigo, como ya queda señalado, la Comunidad de Madrid. En efecto, en un contexto de aumento de las uniones consensuales en el que los nacimientos fuera del matrimonio son cada día más numerosos, centrarse exclusivamente en la fecundidad de los matrimonios es ignorar una parte cada vez más relevante del proceso de la reproducción. Tratar de establecer en qué medida la caída de la nupcialidad ha deprimido la fecundidad matrimonial sin tener en cuenta que muchas mujeres que en la década de los años setenta se casaban, ahora se reproducen emparejadas sin haber pasado por el matrimonio, sería poco menos que ocioso. Es obvio que esas mujeres no han encontrado dificultades para emparejarse $(o$, al menos, no las mismas dificultades que aquellas otras que no viven en pareja): si no están casadas es porque han optado por la cohabitación, hecho que hay que tener presente cuando lo que se persigue es evaluar cómo las trabas al emparejamiento han hecho disminuir el nivel de la fecundidad.

Para sortear este tipo de obstáculos, sugiero, presento y finalmente realizo una nueva descomposición del índice sintético de fecundidad que se basa en la idea de separar los nacimientos de madre casada de los que se producen fuera del matrimonio. El primer paso de este segundo procedimiento consiste en separar la fecundidad total observada en fecundidad matrimonial y fecundidad extramatri-

${ }^{9}$ Una solución a este problema es sustituir el matrimonio por la actividad sexual (STOVER, 1998), aunque los datos sobre las proporciones de mujeres sexualmente activas son mucho más difíciles de obtener que sobre el estado civil. 
monial. En un segundo momento, la fecundidad de los matrimonios se descompone a su vez en los dos factores de interés: proporción de mujeres casadas y nacimientos habidos en los matrimonios; por último, se procede de análoga forma con la fecundidad extramatrimonial, descomponiéndola en la proporción de mujeres no casadas y nacimientos fuera del matrimonio.

El procedimiento de descomposición es sumamente sencillo, a la vez que fácil e intuitivamente comprensible; tiene, además, la ventaja de ser relativamente poco exigente con la información que requiere. Su desarrollo se puede formular como sigue. Sea el ISF la suma de las tasas específicas por edad de fecundidad:

$$
I S F=\sum_{x=15}^{x=49} f(x)=\sum_{x=15}^{x=49} \frac{N(x)}{M(x)}
$$

donde $f(x)$ son las tasas específicas por edad de fecundidad, $N(x)$ el número de nacimientos a la edad $x$ de la madre y $M(x)$ el número de mujeres de edad $x$ en un año dado. Se computa, a continuación, el número total de nacimientos como la suma de los nacimientos de madre casada $N_{c}$ y de madre no casada $N_{n c}$, separando así la fecundidad matrimonial de la extramatrimonial:

$$
I S F=\sum_{x=15}^{x=49} \frac{N_{c}(x)+N_{n c}(x)}{M(x)}=\left[\sum_{x=15}^{x=49} \frac{N_{c}(x)}{M(x)}\right]+\left[\sum_{x=15}^{x=49} \frac{N_{n c}(x)}{M(x)}\right] .
$$

Se descompone después la fecundidad matrimonial en dos factores: uno que expresa la intensidad del comportamiento reproductivo de los matrimonios, indicado por el término $N_{c}(x) / C(x)$; y otro que expresa la intensidad del emparejamiento, indicado por el término $C(x) / M(x)$, que no es otra cosa que la proporción de mujeres casadas a diferentes edades. La descomposición de la fecundidad matrimonial se realiza entonces siguiendo la formula:

$$
\sum_{x=15}^{x=49} \frac{N_{c}(x)}{M(x)}=\sum_{x=15}^{x=49}\left[\frac{N_{c}(x) C(x)}{M(x) C(x)}\right]=\sum_{x=15}^{x=49}\left[\frac{N_{c}(x)}{C(x)} \times \frac{C(x)}{M(x)}\right] .
$$

Análogamente se procede con la fecundidad extramatrimonial, ${ }^{10}$ aislando los dos factores que la integran: de un lado, la intensidad del comportamiento reproductivo fuera del matrimonio, indicado por el término $N_{n c}(x) / N C(x)$; de otro, un

${ }^{10}$ Lo más oportuno sería descomponer la fecundidad extramatrimonial para los diferentes estados civiles de las madres. Debido a la falta de datos, la descomposición se reduce a los estados de casada y no casada. 
segundo factor que expresa otra vez, de forma invertida, la intensidad del emparejamiento, indicado por el término $N C(x) / M(x)$, que no es otra cosa que la proporción de mujeres no casadas a diferentes edades. Por lo tanto, para descomponer la fecundidad extramatrimonial se sigue la fórmula siguiente:

$$
\sum_{x=15}^{x=49} \frac{N_{n c}(x)}{M(x)}=\sum_{x=15}^{x=49}\left[\frac{N_{n c}(x) N C(x)}{M(x) N C(x)}\right]=\sum_{x=15}^{x=49}\left[\frac{N_{n c}(x)}{N C(x)} \times \frac{N C(x)}{M(x)}\right]
$$

Obviamente, la suma de los dos términos descompuestos - fecundidad de los matrimonios y fecundidad fuera de los matrimonios- es igual al índice sintético de fecundidad:

$$
I S F=\sum_{x=15}^{x=49}\left[\frac{N_{c}(x)}{C(x)} \times \frac{C(x)}{M(x)}\right]+\sum_{x=15}^{x=49}\left[\frac{N_{n c}(x)}{N C(x)} \times \frac{N C(x)}{M(x)}\right] .
$$

Una vez realizada la descomposición, se puede interpretar la evolución en el tiempo del ISF como el producto de la variación independiente de nuestros dos factores de interés, comprobando para cada uno de los dos tipos de fecundidad (matrimonial y no matrimonial) cuál de esos dos factores cambia más y verificando qué parte del cambio conjunto corresponde a cada uno de ellos. A esos efectos, y dado lo tedioso que resultaría comparar el gran número de tasas involucradas en el índice agregado, lo más recomendable es simular la evolución que eventualmente habría seguido la fecundidad coyuntural en cada periodo si se hubiera mantenido constante a lo largo del tiempo uno de los dos factores y hubiera variado el otro. Ello permite calcular, para cada uno de los años de referencia, un índice de fecundidad esperado de fácil e inmediata comparación con el índice realmente observado.

Como el objetivo de este ejercicio es evaluar el impacto de la creciente soltería en la fecundidad de las madrileñas, todo invita a mantener constantes las proporciones por edad de casadas y no casadas del año 1975, y determinar qué hubiera ocurrido con la fecundidad en los años posteriores de haber variado sólo las tasas de fecundidad y no la proporción de mujeres emparejadas. El índice de fecundidad esperado con nupcialidad constante en 1975 , ISF ${ }_{1975}$ se calcularía entonces de acuerdo con la siguiente fórmula:

$$
I S F_{1975}^{*}=\sum_{x=15}^{x=49}\left[\frac{N_{c}(x)}{C(x)} \times \frac{C_{1975}(x)}{M_{1975}(x)}\right]+\sum_{x=15}^{x=49}\left[\frac{N_{n c}(x)}{N C(x)} \times \frac{N C_{1975}(x)}{M_{1975}(x)}\right]
$$

donde $C_{1975}$ y $N C_{1975}$ son, respectivamente, las mujeres casadas y no casadas en 1975 , y $M_{1975}$ es el número total de mujeres en ese mismo año. La simulación permite entonces sopesar la influencia del emparejamiento en la fecundidad de 
las mujeres madrileñas y estimar hasta qué punto su creciente soltería ha hecho caer la fecundidad coyuntural hasta los niveles observados en los años noventa.

Dos precisiones se imponen antes de presentar datos y resultados. En primer lugar, a los efectos de este ejercicio, el emparejamiento se operacionaliza como la suma de mujeres casadas y convivientes en uniones consensuales, dos fenómenos que han experimentado una evolución divergente en el periodo estudiado, en el que han disminuido los matrimonios y aumentado las parejas de hecho. Sabemos también que en estos años el crecimiento de la cohabitación, aunque considerable, no ha compensado la caída del número de matrimonios, por lo que la proporción conjunta de mujeres en pareja (casadas más cohabitantes) ha disminuido entre 1975 y 1996. Por lo tanto, cuando se mantienen constantes las proporciones de casadas y no casadas del año 1975 se simula a la perfección la intensidad del emparejamiento en esa fecha, pero no su estructura, es decir, no se tiene en cuenta el cambio en la composición interna (casadas y cohabitantes) del emparejamiento. La dificultad deriva de la posible fecundidad diferencial de ambos tipos de parejas y la solución ideal consistiría en descomponer el índice en tres factores (proporción de mujeres casadas, proporción de mujeres cohabitantes y proporción de mujeres no emparejadas) a los que se debería aplicar, tras mantenerlos constantes en los valores de 1975, sus respectivas fecundidades observadas en los años sucesivos. Por desgracia, no se dispone de la información adecuada para hacerlo, con lo que a esa parte de las casadas de 1975 que en la simulación representan a cohabitantes de los años posteriores se les está aplicando la fecundidad matrimonial de cada año, no la fecundidad de las uniones consensuales, que es la que les correspondería. Una solución aproximada para resolver el problema no revela diferencias sustanciales con los resultados que se presentan aquí ${ }^{11}$.

En segundo lugar, la simulación permite comparar una medida contrafáctica (el ISF esperado) con otra realmente observada (el ISF observado) y, por lo tanto, admite en principio diferentes interpretaciones del sentido de la conexión empírica que se establece entre emparejamiento y fecundidad. En pocas palabras, hay ocasiones en las que es la fecundidad la que precede e incluso produce el emparejamiento, aunque son en verdad tan escasas que no desvirtúan el argumento propuesto. Los casos de interés, que pertenecen al ámbito de la fecundidad extra-

11 La solución alternativa consiste en 1 ) utilizar los nacimientos extramatrimoniales de cada año posterior a 1975 como una aproximación a las mujeres cohabitantes; 2) disminuir para cada edad y año el número de casadas de 1975 en una cantidad equivalente a las cohabitantes (nacimientos de madre no casada) de cada fecha posterior; y 3 ) aumentar el número de mujeres no casadas de 1975 en un número equivalente al de las cohabitantes (nacimientos de madre no casada) de fechas posteriores. Formalmente, el índice sintético de fecundidad esperado se calcularía entonces:

$$
I S F_{1975}^{*}=\sum_{x=15}^{x=49}\left[\frac{N_{c}(x)}{C(x)} \times \frac{\left(C_{1975}(x)-N_{n c}(x)\right)}{M_{1975}(x)}\right]+\sum_{x=15}^{x=49}\left[\frac{N_{n c}(x)}{N C(x)} \times \frac{\left(N C_{1975}(x)+N_{n n}(x)\right)}{M_{1975}(x)}\right]
$$

Como los resultados de esta nueva forma de abordar la descomposición apenas difieren de los que resultan de mantener constante la proporción de casadas de 1975, serán estos últimos los que, debido a su mayor simplicidad conceptual, se presentarán en el texto. 
matrimonial, son los dos siguientes: 1) las mujeres que se reproducen solas ${ }^{12}$, una situación muy excepcional por lo que sabemos de las estructuras familiares en Madrid (en 1991 no más de un 0,5\% de las mujeres entre 15 y 49 años eran madres solteras que vivían solas con hijos a su cargo; véase Requena, 1993); y 2) aquellas de entre las llamadas concepciones prenupciales -el hijo nace durante los ocho primeros meses del matrimonio- que no arrancan de una situación de previo emparejamiento (lo que excluye a los cohabitantes que se deciden por el matrimonio una vez que hay embarazo de por medio), un fenómeno que está en abierto retroceso en nuestro país desde comienzo de los años ochenta ${ }^{13}$.

\section{DATOS}

La información con que se desarrolla el argumento que conecta fecundidad y emparejamiento -así como el doble ejercicio de descomposición del índice sintético de fecundidad en que se apoya- procede, como es de esperar, de las dos fuentes canónicas que típicamente registran los datos de flujos y de stock y con las que habitualmente se calcula el ISF. Por una parte, los registros vitales (las estadísticas del movimiento natural de la población) documentan los eventos de interés, en nuestro caso los nacimientos según la edad de la madre, que constituyen los numeradores de las tasas específicas de fecundidad; por otra, los censos de población y los padrones municipales suministran para cada año de referencia las poblaciones expuestas al riesgo de experimentar los mencionados eventos de interés, que se convierten en los denominadores de dichas tasas. En el Anexo de Datos se presentan las distribuciones de mujeres y nacimientos por edad y estado civil de la madre en la Comunidad de Madrid (circunscripción territorial antes denominada provincia de Madrid) para los años 1975, 1981, 1986, 1991 y 1996.

Como se ha señalado más arriba, el ejercicio que aquí presento se construye con datos relativos a Madrid. Esta opción, que en apariencia limitaría innecesariamente el alcance de las conclusiones, exige una pequeña explicación. La razón de que el presente ejercicio quede acotado a la comunidad madrileña obedece simple y llanamente a un problema de disponibilidad de los datos de base. En efecto, una de las ventajas de las descomposiciones del índice sintético de fecundidad que acometo es que no resultan en exceso exigentes con la información requerida; pero para llevarlas a cabo forzosamente hay que contar, en lo que se refiere a los datos de flujos, con a) la distribución de los nacimientos por edad y estado civil de la madre, lo que permite computar los numeradores de las tasas; $y$, en lo que se refiere a los datos de

12 En el otro tipo de nacimientos extramatrimoniales - los hijos de las parejas cohabitantes- no se plantea el problema del sentido de la conexión entre emparejamiento y fecundidad.

${ }_{13}$ En palabras de un experto: «... este tipo de concepción [concepción seguida rápidamente de boda] correspondía frecuentemente a parejas ya formadas, muchas de ellas con un proyecto matrimonial preciso, mientras que en los casos de boda tardía se trataba más bien de matrimonios que, sin el embarazo, no se hubieran celebrado». (MuÑoz, 1995, 203). En todo caso, cabe añadir que muy buena parte de las concepciones prenupciales se concentran en edades inferiores a los 22 años, cuya fecundidad contribuye escasa y decrecientemente a la fecundidad total. 
stock, que nos proporcionan los denominadores de las tasas, con $b$ ) la distribución de la población femenina por estado civil en los años de referencia.

Como se sabe, las estadísticas del movimiento natural de la población suministran el número de nacimientos que se produce cada año en España según la edad y el estado civil de la madre ${ }^{14}$. Por su parte, censos y padrones proporcionan las distribuciones de la población femenina española por sexo, edad y estado civil en los años de referencia de 1975, 1981, 1986 y 1991, aunque no de 1996. Esta carencia se debe a la peculiar política de recogida de información aplicada con ocasión de los padrones municipales de habitantes de 1996. Es el caso que en la operación padronal correspondiente a esa última fecha no todos los municipios españoles utilizaron cuestionarios que recogieran el estado civil de los habitantes y, en consecuencia, no fue posible registrar la información referida a esa fundamental variable a escala nacional. El resultado de esa política, lamentable por muchos conceptos, ha sido el de generar una pérdida irreparable en la información estadística de nuestro país: al día de hoy, no se cuenta con una fuente de datos que, basada en una encuesta total, registre en el ámbito nacional la distribución de la población española por estado civil en el año 1996.

Puesto que no se dispone de esa información básica, no es posible realizar el ejercicio propuesto a escala nacional en ese año de 1996 con las garantías que ofrece trabajar con datos que proceden del mismo tipo de fuente. Una posible solución a esa falta de datos nacionales podría consistir en utilizar información extraída de encuestas muestrales; por ejemplo, de la Encuesta de Poblacion Activa que sería, dada su muy amplia base muestral y su gran periodicidad, una obvia candidata a sustituir al incompleto, y a nuestros efectos inservible, padrón de 1996 en el cómputo y la descomposición de las tasas correspondientes a ese año. Sin embargo, siendo el cálculo de las tasas específicas por edad de fecundidad muy sensible a los denominadores utilizados, no parece lo más recomendable introducir en su cómputo un elemento adicional de heterogeneidad acudiendo a una fuente como la EPA que presenta acreditadas divergencias con respecto a los censos que no son en modo alguno despreciables ${ }^{15}$. La solucion por la que he optado ha sido la de circunscribir datos y resultados a la comunidad madrileña, en cuyos municipios sí se registro el estado civil de sus habitantes en 1996 y para la que sí que se cuenta con una serie completa de datos relativos a la edad y estado civil de sus habitantes en cada una de las fechas de interés.

Limitar el alcance de este ejercicio a la comunidad madrileña plantea algunos problemas; pero también ventajas ciertas que no deben pasarse por alto. En cuanto a los problemas, tal vez el principal sea el limitado alcance de los resultados que se obtienen al estudiar un universo demográfico que, al final del periodo considerado, apenas sobrepasa los cinco millones de personas. Es cierto que la evolución de los indicadores básicos de la fecundidad coyuntural en Madrid presenta un gran paralelismo con la que ha seguido el conjunto de España (véase

14 Distinguiendo, al menos, los nacimientos de las madres casadas de los que corresponden a madres no casadas, una desagregación que resulta suficiente para nuestros propósitos.

15 Para las discrepancias entre los datos de los censos y la EPA, véanse REQUENA (1999) y FERNÁNDEZ CORDÓN (2001). 
gráfico 3), pero la existencia en nuestro país de diferentes regímenes reproductivos en sus distintas zonas geográficas es innegable (Gil y Cabré, 1997): País Vasco, Cantabria, Galicia y Castilla León se sitúan en el extremo menos fecundo; Murcia, Extremadura, Andalucía, Baleares, Castilla La Mancha y Canarias caen en el polo opuesto de máxima fecundidad; la Comunidad de Madrid, junto con Cataluña, ha venido ocupando una posición intermedia entre ambos límites, con una fecundidad instantánea ligeramente inferior al promedio nacional.

GrÁFICO 4

Evolución del índice sintético de fecundidad en España y Madrid

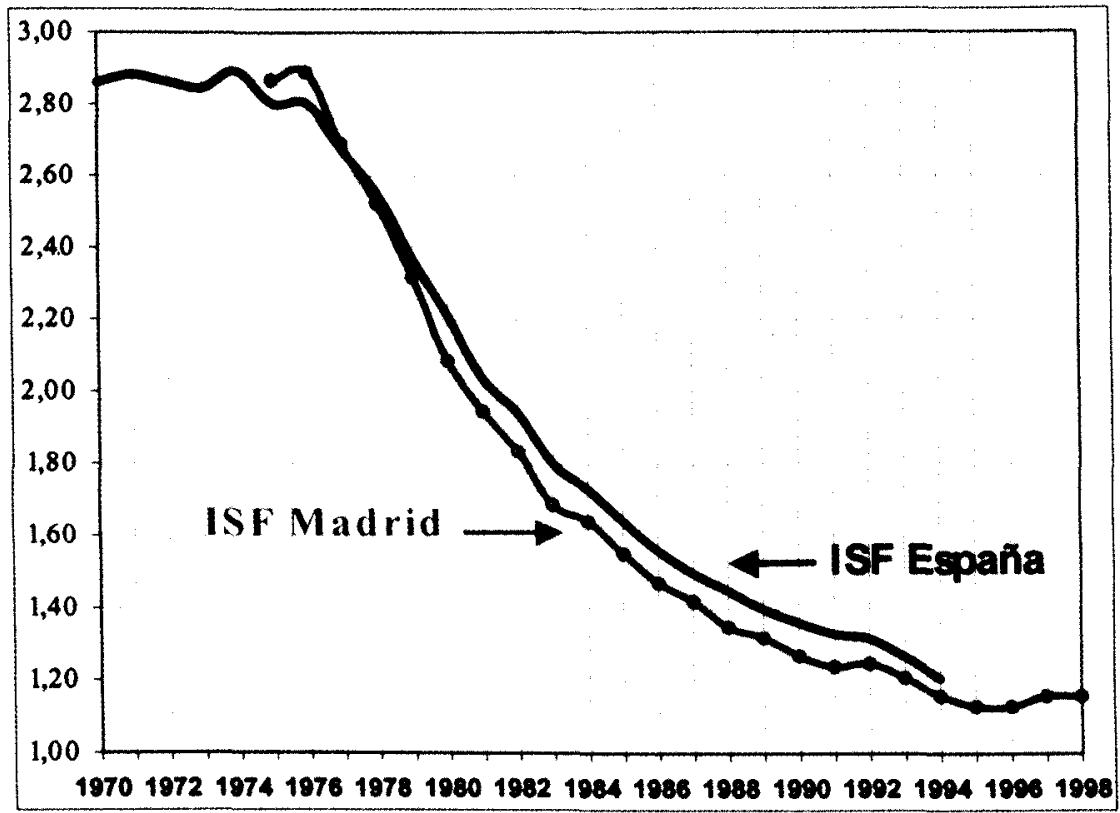

Fuente: Instituto Nacional de Estadística y Comunidad de Madrid.

Como es lógico, toda esa diversidad regional subyacente a los promedios nacionales impide que las conclusiones relativas a una determinada zona -la comunidad madrileña en este caso- sean automáticamente extrapolables o generalizables a escala de todo el país. En ningún momento se pretende tal cosa. Con todo, no hay que ignorar el provecho que se obtiene de trabajar con información relativa a un ámbito poblacional que contiene menos heterogeneidad interna que el conjunto nacional, base de no pocos análisis demográficos al uso. $\mathrm{Al}$ acotar datos y argumentos a sólo una comunidad autónoma, el riesgo de incurrir en falacias de composición derivadas del análisis de promedios correspondientes a poblaciones con una gran variedad interna disminuye de forma importante. Disminución que resulta aún más oportuna cuando, como es el caso presente, la información empírica sobre la que se trabaja no consiste en microdatos recogidos a escala de los individuos, sino en tasas calculadas sobre agregados. 
Por lo demás, las particulares características de la Comunidad de Madrid, con una estructura social y económica plenamente modernizada y con un comportamiento demográfico coherente con las pautas dominantes en la Europa del sur (Requena, 1993), suponen a priori una cierta garantía de coherencia e interés de los resultados. Es de esperar, por ello, que las conclusiones que se extraen del análisis de la evolución de la fecundidad en la Comunidad de Madrid sí sean representativas de esas sociedades del sur de Europa que hoy experimentan un régimen de muy baja fecundidad.

\section{RESULTADOS}

La descomposición de la tasa de fecundidad total (TFR) realizada siguiendo el método propuesto por Bongaarts (tabla 1 y gráfico 4) pone de manifiesto dos fases y dos pautas claramente diferenciadas en la disminución de la fecundidad que han experimentado las mujeres residentes en Madrid entre 1975 y 1996. La primera fase, que se extiende entre 1975 y 1981, se caracteriza por una intensa caída de la fecundidad (desde 2,8 hijos por mujer hasta 1,9; la magnitud del índice disminuye en casi una tercera parte) concentrada en un lapso muy corto de tiempo (seis años). Esta caída se explica en su casi totalidad por el descenso de la fecundidad matrimonial. Nótese que la fecundidad matrimonial (TMFR, la fecundidad que se podría esperar que se diera si el matrimonio no pusiera trabas a la procreación) disminuye en la misma medida que la fecundidad total entre 1975 y 1981, desde un máximo de 5,9 hijos por mujer hasta 4,2. El índice de matrimonio apenas varía en esos seis años, lo que significa que el celibato apenas puso en 1981 más restricciones a la fecundidad total que las que ya ponía en 1975 (una coyuntura de máxima nupcialidad). Dicho en otros términos, no habiendo prácticamente incidido el celibato en la fecundidad entre 1975 y 1981 , el fuerte descenso de esta última se puede imputar al cambio en los comportamientos reproductivos de los matrimonios, que controlaron su natalidad con más intensidad que antes dejando que actuaran los factores inhibidores relacionados con los métodos anticonceptivos.

\section{TABLA 1}

Descomposición de la TFR según el método de Bongaarts

\begin{tabular}{|c|c|c|c|c|c|}
\hline & 1975 & 1981 & 1986 & 1991 & 1996 \\
\hline TFR observada ............ & 2,841 & 1,954 & 1,481 & 1,247 & 1,137 \\
\hline TMFR ............................ & 5,907 & 4,240 & 4,172 & 3,786 & 4,073 \\
\hline $\mathrm{Cm}$ & 0,48 & 0,46 & 0,36 & 0,33 & 0,28 \\
\hline TFR esperada ................ & 2,841 & 2,039 & 2,006 & 1,821 & 1,959 \\
\hline
\end{tabular}

Fuente: elaboración propia a partir de los datos del anexo.

Desde 1981 hasta 1996 la fecundidad coyuntural de las madrileñas sigue cayendo, aunque a un ritmo menor que en los seis años precedentes: en ese periodo el ISF pasa de 1,9 hijos por mujer a sólo 1,1, una caída de un $40 \%$ en el valor del índice pero repartida a lo largo de tres lustros. Sin embargo, lo importante es que la pauta de descenso de la fecundidad es radicalmente distinta a la de los seis años anterio- 
res, pues su variación obedece casi totalmente al impacto de la creciente soltería sobre la capacidad reproductiva de las madrileñas. Mientras la fecundidad total observada cae hasta alcanzar mínimos históricos, la fecundidad matrimonial estimada se instala en 1981 en el entorno de los 4 hijos por mujer, un nivel que ya no abandona hasta 1996 (con una leve caída en 1991 que, no obstante, parece haberse recuperado en 1996). Como es logico, el índice de matrimonio cae hasta situarse en su nivel más bajo en 1996, lo que sugiere que es la decreciente proporción de mujeres casadas lo que ha deprimido la fecundidad observada en ese periodo.

Por lo tanto, recurriendo al lenguaje de los determinantes próximos de la fecundidad, se puede decir que entre 1975 y 1981 fueron sobre todo los factores relacionados con el control deliberado de la natalidad dentro de los matrimonios -además, obviamente, de los factores específicamente limitadores de la fertilidad natural- los responsables de la depresión de la fecundidad, que cae a un ritmo muy intenso. Entre 1981 y 1996 se puede interpretar, en cambio, que las variables intermedias relativas al control de los nacimientos en el seno de las parejas y las restricciones a la fertilidad natural no tuvieron apenas mayor incidencia de la que habían tenido entre 1975 y 1981. Esto, al menos, es lo que se deduce del hecho de que los matrimonios mantuvieran su fecundidad estimada en niveles muy similares durante esos quince años. De hecho, si se hubiera mantenido estable el índice matrimonial de 1975 , se podría haber esperado que la fecundidad coyuntural que se hubiera dado en 1981 no fuera muy diferente de la que realmente se produjo; en cambio en 1986, 1991 y 1996, la fecundidad observada cae y se aleja de la fecundidad esperada, que se mantiene en un nivel muy próximo a los dos hijos por mujer.

Gráfico 5

Descomposición de la TFR de Madrid según el método de Bongaarts

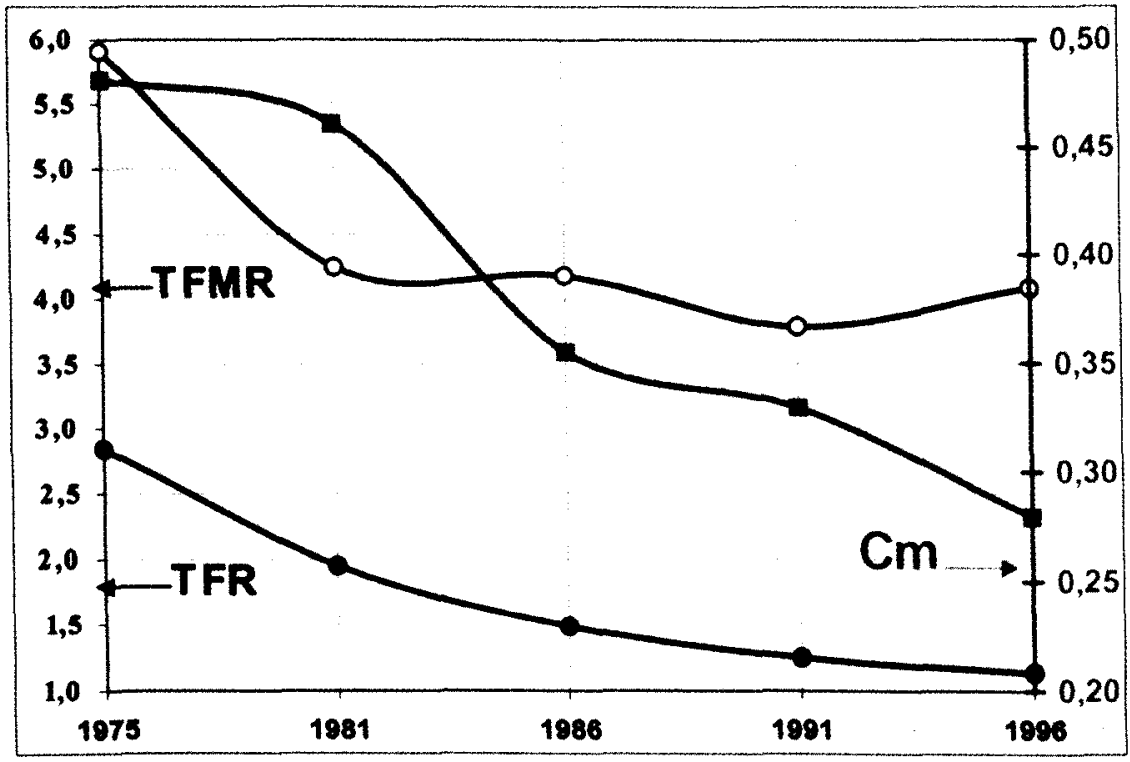

Fuente: Elaboración propia a partir de los datos del anexo. 
La descomposición del ISF propuesta a partir de la fecundidad matrimonial y la extramatrimonial conduce a conclusiones muy similares (tabla 2). En este segundo caso, el procedimiento de estandarización de las tasas consiste en mantener constante no ya la nupcialidad, sino el nivel de emparejamiento (mujeres casadas y no casadas) de 1975; y el instrumento básico de interpretación de los resultados es la comparación entre el ISF esperado si no se hubiera alterado la intensidad de emparejamiento de 1975 y el realmente observado en cada año.

Con este método alternativo se pueden advertir, de nuevo, dos formas de descenso de la fecundidad a lo largo del periodo, que corresponden a las dos fases y pautas que ya se perfilan con la descomposición realizada por el método de Bongaarts. Entre 1975 y 1981 el grueso de la disminución se puede atribuir a la fecundidad de las mujeres emparejadas: de hecho, si en 1981 se hubieran mantenido constantes los niveles de emparejamiento de 1975, la fecundidad total esperada apenas hubiera diferido de la realmente observada (ambas muy próximas a los dos hijos por mujer). Lo que significa dos cosas: 1) que el descenso de la fecundidad de finales de los años setenta no se puede imputar a una caída en la intensidad del emparejamiento; y 2) que, por lo tanto, hay que pensar más bien en que la caída de la fecundidad se alcanzó porque las parejas controlaron más su capacidad reproductiva.

\section{TABLA 2}

Descomposición del ISF en fecundidad matrimonial y extramatrimonial

\begin{tabular}{lccccc}
\hline & 1975 & 1981 & 1986 & 1991 & 1996 \\
\hline ISF observado ........... & 2,841 & 1,954 & 1,481 & 1,247 & 1,137 \\
\hline ISF* casadas.............. & 2,765 & 1,912 & 1,750 & 1,611 & 1,685 \\
\hline ISF* no casadas .......... & 0,075 & 0,094 & 0,098 & 0,096 & 0,081 \\
\hline ISF* esperado............ & 2,841 & 2,006 & 1,848 & 1,707 & 1,766 \\
\hline
\end{tabular}

Fuente: elaboración propia a partir de los datos del anexo.

A partir de 1981 y hasta 1996 la fecundidad observada cae mucho más intensamente que la fecundidad esperada (gráfico 6). Esto indica un significativo cambio de pauta, y por tanto, exige una interpretación muy distinta. Si los niveles de emparejamiento de 1975 se hubieran mantenido constantes en los años posteriores, la fecundidad esperada habría disminuido hasta aproximadamente 2 hijos por mujer en 1981 (como sucedió en realidad con la observada) para situarse a partir de 1986 en el entorno de los 1,8 hijos por mujer, en el que prácticamente se mantuvo hasta 1996. Una gran parte de la caída de la fecundidad acaecida entre 1981 y 1996 hay que interpretarla entonces como una consecuencia de la disminución de la intensidad del emparejamiento de las madrileñas en ese periodo: mientras la fecundidad esperada mantuvo un nivel de relativa estabilidad en torno a los 1,8 hijos por mujer, la fecundidad observada se fue hundiendo hasta situarse en los niveles ínfimos correspondientes a 1996. La estabilidad de la fecundidad esperada desde 1981 lleva a explicar el cambio de la observada como un producto del descenso en los niveles de emparejamiento de las mujeres madrileñas. A efectos de 
cuantificación, se podría decir que el impacto del menor nivel de emparejamiento de las madrileñas explica en torno a tres cuartas partes de la disminución de la fecundidad coyuntural en 1986, 1991 y 1996 respecto a la observada en 1981.

GráfICO 6

ISF de Madrid observado y esperado con nupcialidad de 1975 constante

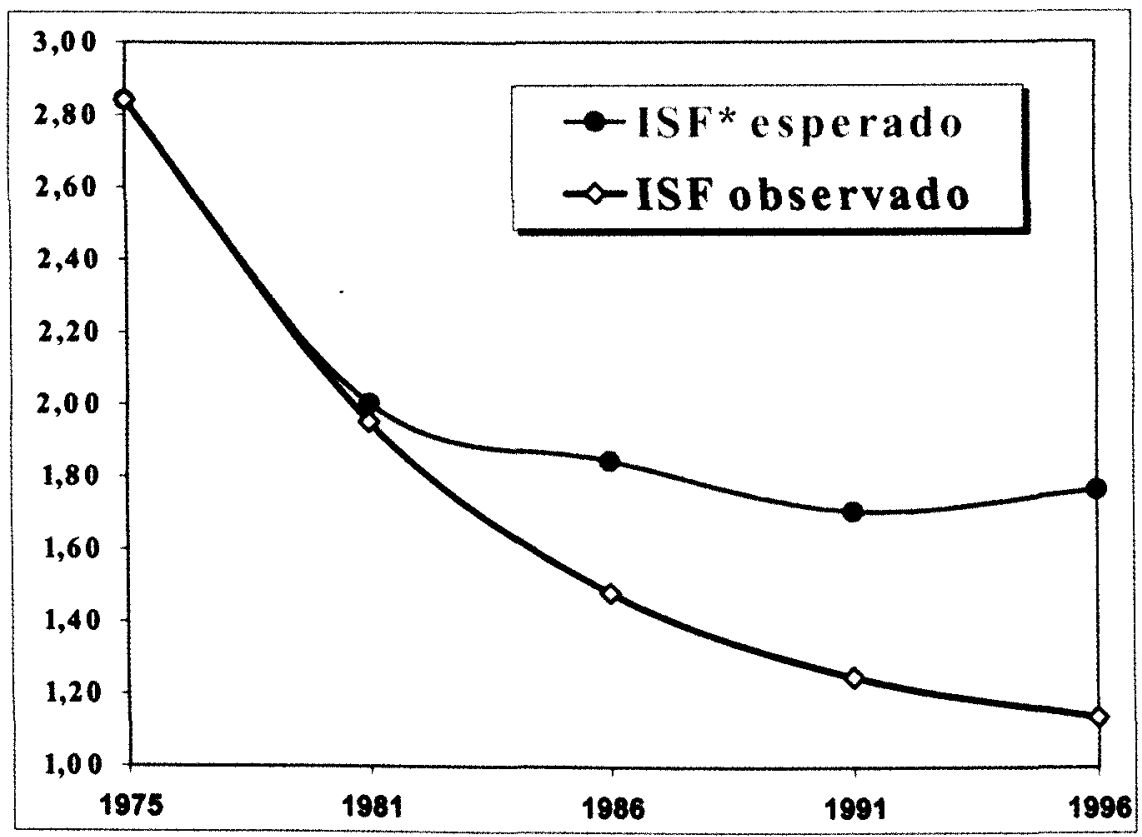

Fuente: elaboración propia a partir de los datos del anexo.

Los resultados de los dos ejercicios de descomposición del ISF de las madrileñas entre 1975 y 1996 conducen a una misma interpretación de la reciente e intensa caída de la fecundidad en esta comunidad. Entre 1975 y 1981 se observa una caída muy acentuada de la fecundidad coyuntural que sólo se explica por el mayor esfuerzo anticonceptivo de las parejas. A partir de esa fecha, las parejas mantienen seguramente un patrón de anticoncepción muy similar al de los años anteriores, con la diferencia que el stock de mujeres emparejadas en disposición de procrear fue disminuyendo en términos relativos (y también en términos absolutos desde 1981), simplemente porque el ritmo de formación de nuevos matrimonios y parejas se desaceleró de manera extrema.

Hasta comienzos de los años ochenta, por tanto, tres características perfilan la coyuntura demográfica de la Comunidad de Madrid: nupcialidad intensa, matrimonio precoz y fecundidad decreciente apoyada en el uso generalizado de la anticoncepción por parte de las parejas. Estas son precisamente las características que cabe esperar de una comunidad que se sitúa al final de la transición de la fecundidad: una estructura nupcial muy poco restrictiva (mucha gente se empa- 
reja y lo hace a una edad relativamente temprana) con una fecundidad de momento sólo ligeramente por debajo de los niveles de reemplazo generacional. Sin embargo, durante los años ochenta y noventa se puede observar un cambio relevante, y hasta cierto punto imprevisto, de los comportamientos de emparejamiento y reproducción en las madrileñas que da lugar a una nuevo patrón demográfico. ¿En qué consiste ese nuevo patrón? En primer lugar, la nupcialidad se deprime extraordinariamente, con una relevante disminución del número de mujeres emparejadas en edad fértil (o, visto en perspectiva longitudinal, con una disminución muy considerable del tiempo de su ciclo vital fértil durante el que están emparejadas). En segundo lugar, la caída de la nupcialidad es de tal magnitud que no se ve compensada por la formación de parejas de hecho que, en todo caso, es un fenómeno creciente. Tercero, la entrada a la vida en pareja, ya sea matrimonio o cohabitación, se retrasa de forma sustancial. Cuarto, la fecundidad de las parejas que sí se han formado se mantiene en niveles muy parecidos a los de los primeros años ochenta. Por último, el impacto de la cada vez más escasa y tardía nupcialidad en la fecundidad ya controlada de las pocas parejas que se han formado deprime los niveles reproductivos coyunturales por debajo del umbral de la muy baja fecundidad. Este conjunto de factores es el que parece definir el régimen de muy baja fecundidad, por debajo de 1,3 hijos por mujer, que tipifica en nuestros días a algunas sociedades del sur de Europa, como es el caso de la Comunidad de Madrid.

\section{CONCLUSIONES}

Es bien conocido que todas las sociedades demográficamente avanzadas han venido reduciendo la intensidad de sus comportamientos reproductivos a medida que realizaban la transición de la fecundidad. A lo largo de ese proceso el nivel de la fecundidad ha experimentado en esas sociedades una muy notable disminución, hasta un punto en que hoy parece difícil imaginar escenarios sociales, económicos o culturales en los que las mujeres de ese tipo de sociedades vayan a tener al final de sus vidas un promedio de hijos que exceda significativamente el número de dos. Muchas proyecciones demográficas suponen, de hecho, para las poblaciones postransicionales una fecundidad próxima al reemplazo generacional, aunque no falta quien sostiene de forma convincente (por ejemplo, Demeny, 1997) que el desenlace reproductivo de la transición demográfica consistirá en sociedades que se situarán claramente por debajo del nivel de substitución de las generaciones. Sea como fuere, algunos países -y algunas zonas dentro de ellos- han ido ya tan lejos en ese proceso que en los últimos años han terminado por incorporarse a un régimen que se califica como de muy baja fecundidad. Tal es el caso de Italia, Grecia y España, y muy señaladamente el de la Comunidad de Madrid, que en 1998 registraba un índice sintético de fecundidad de 1,16 hijos por mujer.

¿Qué mecanismos han impulsado ese acusadísimo declive reproductivo de las mujeres madrileñas hasta los niveles actuales de la muy baja fecundidad? Aunque en este trabajo no se pretende elaborar una interpretación exhaustiva de los hechos, de los resultados de la descomposición de la fecundidad coyuntural rea- 
lizada se sigue que el periodo que media entre 1975 y 1996 puede dividirse en dos partes: durante la segunda mitad de los años setenta, virtualmente todo el descenso de la fecundidad que efectivamente se produjo se puede atribuir a los mayores esfuerzos contraceptivos de los matrimonios y las parejas convivientes; sin embargo, a partir de 1981, y al menos hasta 1996, han sido sobre todo las restricciones a la nupcialidad y el emparejamiento las responsables de la intensísima caída de los niveles coyunturales de la fecundidad que se ha podido observar en la Comunidad de Madrid. En los términos contrafácticos en los que se apoya este ejercicio, ${ }^{16}$ se puede decir que si la intensidad del emparejamiento de las mujeres madrileñas se hubiera mantenido en las tasas observadas en los años setenta, la fecundidad coyuntural se habría situado a lo largo de los años ochenta y primeros noventa en torno a los 1,8 hijos por mujer.

Este resultado es perfectamente coherente con la idea de una transicion hacia el aplazamiento de la fecundidad que, siendo característica de muchas poblaciones desarrolladas, habría comenzado en España en 1979 (Köhler, Billari y Ortega, 2001); sin embargo, debe ser en alguna medida sorprendente para quienes piensan que uno de los principales logros de la modemización demográfica es haber separado la «pasión entre los sexos» de la función reproductiva (Van de Walle, 1983). El uso generalizado de métodos anticonceptivos cómodos, seguros y fiables habría acabado con la función tradicional del matrimonio y la formación de la pareja como mecanismo homeostático de control de la reproducción. Pero, contra lo que cabía esperar, la disolución de la pauta europea de matrimonio no ha dejado paso a una situación en la que se minimizan las restricciones preventivas al emparejamiento y la formación de nuevas unidades familiares. Antes bien, los frenos preventivos han regresado en estas sociedades al primer plano. Hay, por eso, en todo este proceso una suerte de ironía maltusiana: pues han sido las'crecientes dificultades para emparejarse y constituir nuevas familias las que, actuando sobre una capacidad reproductiva ya controlada por los métodos al uso en las poblaciones postransicionales, las que significativamente han deprimido la fecundidad hasta los niveles actuales.

Cabe establecer una última precisión sobre las implicaciones prácticas de este trabajo. El interés del ejercicio que aquí presento no se limita, según creo, al conocimiento sustantivo del fenómeno de la fecundidad y de su evolución a la baja durante los últimos años. Sino que tiene también importancia práctica para el diseño de las políticas públicas que se orientan a incentivar la actividad reproductiva de las mujeres en sociedades que, como la española, han terminado por incorporarse a un régimen demográfico de bajísima fecundidad. La necesidad de tales políticas es hoy ampliamente reconocida. Y, de hecho, muy a menudo se defienden políticas familiares que tratan de movilizar a las mujeres casadas o emparejadas para que tengan más hijos, ofreciéndoles subvenciones directas por hijo, exenciones fiscales o financiación pública de los servicios de apoyo a la crianza. Ahora bien, si es cierto que una parte muy importante de la caída de la fecundidad obedece simplemente al retraso en el calendario de los matrimonios y la formación de

${ }^{16}$ Para una muy convincente defensa de los argumentos contrafácticos, véase LIEBERSon (1985, 45 y ss.). 
parejas y a la disminución de su número, parecería más lógico dirigir esas políticas de fomento de la natalidad a aquellos segmentos de la población responsables del retraso y la caída del emparejamiento: es decir, a los jóvenes adultos que viven en condiciones de dependencia prolongada de sus familias de origen. Las conclusiones de este trabajo vienen así a coincidir con el diagnóstico formulado por Cabré (1999) y con el pormenorizado análisis de Delgado (2000). Si convenimos en la necesidad de arbitrar medidas de fomento de la natalidad, las que mayor eficacia alcanzarían serían aquellas que facilitaran a los jóvenes la emancipación de sus familias de origen, potenciando la formación de parejas que conviven de forma independiente y de nuevas unidades familiares.

\section{REFERENCIAS BIBLIOGRÁFICAS}

AgÚERo, Isabel y Alberto Olano (1982): «La intensa caída reciente de la fecundidad y la nupcialidad en España». En Rosa Conde, comp.: Familia y cambio social en España. Madrid: Centro de Investigaciones Sociológicas, pp. 31-61.

BongaARTs, John (1978): «A Framework for Analyzing the Poximate Determinants of Fertility", Population and Development review 4, 1, pp. 105-132.

- (1982): «The Fertility-Inhibiting Effects of the Intermediate Fertility Variables», Studies in Family Planning 13, 6/7, pp. 179-189.

BongaARTs, John y Griffith FeEnEY (1998): «On the Quantum and Tempo of Fertility», Population and Development review 24, 2, pp. 271-291.

CABRE, Anna (1999): El sistema català de reproducció. Barcelona: Institut Català de la Mediterrània d' Estudis i Cooperació.

CASTRO, Teresa (1992): «Delayed childbearing in contemporary Spain: trends and differentials», European Journal of Population, 8, pp. 217-246.

- (1993): «Changing Nuptiality Patterns in contemporary Spain», Genus, vol. II, n $1-2$, pp. 79-95.

CoAle, Ansley (1967): «Factors Associated with the Development of Low Fertility: An Historic Summary». En Proceedings of the United Nations World Population Conference, Belgrade 1965, vol. II. Nueva York: United Nations, pp. 205-209.

- (1986): «The Decline of Fertility in Europe since the Eighteenth Century As a Chapter in Demographic History». En Ansley Coale y Susan C. Watkins, eds., The Decline of Fertility in Europe. Princeton: Princeton University Press, pp. 1-30.

De Miguel, Amando (1991): La población de Madrid a lo largo del último siglo. Madrid: Asamblea de Madrid.

Delgado, Margarita (2000): «Los componentes de la fecundidad: su impacto en la reducción del promedio de hijos por mujer en España», Economistas, 86, pp. 23-34.

Demeny, Paul (1997): «Replacement-Level Fertility: The Implausible Endpoint of Demographic Transition». En Gavin W. Jones, Robert M. Douglas, John C. CALdwell y Rennie M. D'Souza, eds., The Continuing Demographic Transition. Oxford Clarendon Press, pp. 94-110.

Domingo, Andreu (1997): La formación de la pareja en tiempos de crisis. Madrid y Barcelona, 1975-1995. Tesis doctoral. Madrid: Universidad Nacional de Educación a Distancia.

Fernández, Juan A. (1986): «Análisis longitudinal de la fecundidad en España». En Alberto Olano, comp., Tendencias demográficas y planificación económica. Madrid: Ministerio de Economía y Hacienda, pp. 49-75. 
- (1993): La Población y la fecundidad de la Comunidad de Madrid. Informe monográfico del Tomo I de los Censos de Población y vivienda de 1991. Madrid: Comunidad de Madrid, Consejería de Economía.

FERnÁNDEZ, Juan A. (1997): «Youth Residential Independence and Autonomy: A Comparative Study», Journal of Family Issues, 18 (6), pp. 576-607.

FERNÁNDEZ, Juan A., dir. (2001): Hogares y familias en Andalucía. Evolución y proyección hasta 2016. Sevilla: Instituto de Estadística de Andalucía.

GARRIDO, Luis (1993): «La familia estatal: el control fiscal de la natalidad». En Luis GARRIDO y Enrique Gil CALvo, eds., Estrategias familiares. Madrid: Alianza, pp. 157-180.

- (1996): «La revolución reproductiva». En Cecilia Castaño y Santiago Palacios, eds.: Salud, dinero y amor. Cómo viven las mujeres españolas de hoy. Madrid: Alianza, pp. 205-238.

GARRIDO, Luis y Miguel Requena (1987): «Integración socioeconómica en la Comunidad de Madrid». Alfoz, 47, pp. 47-64.

GARRIDO, Luis y Miguel REQuenA (1995): «El acceso de los jóvenes a la vivienda y al trabajo». Revista Asturiana de Economía, 2, pp. 27-54.

GARRIDO, Luis y Miguel Requena (1996): La emancipación de los jóvenes en España. Madrid: Ministerio de Trabajo y Asunto Sociales, Instituto de la Juventud.

GIL, Fernando y Anna CABRE (1997): «El crecimiento natural de la población española y sus determinantes». En Rafael Puyol, ed., Dinámica de la población en España. Cambios demográficos en el último cuarto del siglo XX. Madrid: Síntesis, pp. 47-144.

Grabill, Wilson H., Clyde V. Kiser y Pascal K. Whelpton (1958): The Fertility of American Women. Nueva York: Wiley.

HAJNAL, John (1965): «European marriage pattern in perspective». En David V. GLASS y D.E.C. Eversley, eds.: Population in History. Londres: Arnold, págs. 101-143.

JURADO, Teresa (2001): Youth in Transition. Housing, Employment, and Social Policies in France and Spain. Ashgate: Aldershot.

Köhler, Hans-Peter, Francesco Billari y José Antonio Ortega (2001): «Towards a Theory of Lowest-Low Fertility», MPIDR Working Paper WP 2001-32, Max Plank Institut für demografische Forschung, Rostock.

LiEBERSON, Stanley (1985): Making It Count. The Improvement of Social Research and Theory. Berkeley: University of California Press.

LimanONDA, Bhassorn (1992): «Nuptiality Patterns in Thailand: Their Implications for Further Fertility Decline». En Calvin Goldscheider, ed.: Fertility Transitions, Family Structure and Population Policy. Boulder: Westview, pp. 101-120.

Martínez-Granado, Maite y Javier Ruiz-Castillo (1998): «The Decisions of Spanish Youth: A Cross-Section Study», Working Paper98-42, Departamento de Economía, Universidad Carlos III de Madrid.

MiReT, Pau (2002): La primonupcialidad en España durante el siglo XX. Tesis doctoral. Madrid: Universidad Nacional de Educación a Distancia.

MuÑoz, Francisco (1995): «Procreación y matrimonio en España (1970-1990)», Revista Internacional de Sociología, 11, pp. 197-238.

Ni Bhrolcháin, Maire (1992): «Period Paramount? A Critique of the Cohort Approach to Fertility", Population and Development review 18, 4, pp. 599-629.

PÉREZ DfAZ, Julio (2002): Transformaciones sociodemográficas e los recorridos hacia la madurez. Las generaciones españolas 1906-1945. Tesis doctoral. Madrid: Universidad Nacional de Educación a Distancia.

PuYol, Rafael (1999): «Evolución y cambios en la población». En José L. García Delgado, dir., España: Economía: Ante el siglo XXI. Madrid: Espasa-Calpe, pp. 59-80.

REHER, David S. (1996): La familia en España, pasado y presente. Madrid: Alianza. 
Requena, Miguel (1993): «Desigualdad social y dependencia familiar en España». En I simposio sobre igualdad y distribución de la renta y la riqueza. Volumen V: Estructura social y movilidad. Madrid: Fundación Argentaria, pp. 59-86.

Requena, Miguel (1993): Los hogares y las formas familiares de la Comunidad de Madrid. Informe monográfico del Tomo $V$ de los Censos de Población y Vivienda de 1991. Madrid: Comunidad de Madrid, Consejería de Economía.

- (1997): «Sobre el calendario reproductivo de las mujeres españolas». Revista Española de Investigaciones Sociológicas, 79, pp. 43-79.

- (1999): «Pautas contemporáneas de evolución de los hogares en España», Revista Internacional de Sociología, 22, pp. 33-65.

- (2001): «Los jóvenes españoles de los años noventa: formación, trabajo, convivencia». Revista de Educación, 325, pp. 33-47.

- (2002): «Juventud y dependencia familiar en España». Revista de Estudios de Juventud, [en prensa].

Rowland, Robert (1988): «Sistemas matrimoniales en la Península Ibérica (siglos XVIXIX). Una perspectiva regional». En Vicente PÉREZ MOREDA y David S. ReHER, eds.: Demografía histórica en España. Madrid: El Arquero, pp. 72-137.

StOVER, John (1998): «Revising the Proximate Determinants of Fertility Framework: What Have We Learn in the Past Twenty Years?», Studies in Family Planning 29, 3, pp. 255-267.

VALERO, Ángeles y Carmen LENCE (1995): «Nupcialidad, fecundidad y familia. La paradoja del comportamiento de la nupcialidad y la fecundidad en España», Revista Internacional de Sociología, 11, pp. 89-114.

VAN DE WALLE, Etienne (1978): «La nucialidad y la fecundidad marital». En David V. Glass y Roger Revelle, eds., Población y cambio social. Estudios de demografia histórica. Madrid: Tecnos, pp. 143-156.

- (1983): «Maltuhs today». En Jacques Dupàquier, Antoinette Fauve-Chamoux y Eugene Grebenik, eds., Malthus Past and Present. Londres: Academic Press, pp. 233245.

VAN IMHOFF, Evert y NICO Keilman (2000): «On the Quantum and Tempo of Fertility: Comment», Population and Development review 26, 3, pp. 549-553.

WRIGLEY, E. Anthony (1992): Gentes, ciudades y riqueza. La transformación de la sociedad tradicional. Barcelona: Crítica.

WUNSCH, Guillaume (1982): «Effect of Changes in Nuptiality on Natality in Western Europe». En Lado T. Ruzicka, ed., Nuptiality and Fertility. Lieja: Ordina, pp. 155-74. 
ANEXO DE DATOS

TABLA I

Mujeres y nacimientos de la provincia de Madrid en 1975

\begin{tabular}{|c|c|c|c|c|c|c|}
\hline \multirow{2}{*}{$\begin{array}{l}1975 \\
\text { Edad }\end{array}$} & \multicolumn{3}{|c|}{ Mujeres } & \multicolumn{3}{|c|}{ Nacimientos } \\
\hline & Total & Casadas & No casadas & Total & $\begin{array}{c}\text { De madre } \\
\text { casada }\end{array}$ & $\begin{array}{l}\text { De madre } \\
\text { no casada }\end{array}$ \\
\hline 15 & 35556 & 160 & 35396 & 62 & 52 & 10 \\
\hline 16 & 35389 & 443 & 34946 & 219 & 152 & 67 \\
\hline 17 & 34944 & 860 & 34084 & 533 & 453 & 80 \\
\hline 18 & 35348 & 1644 & 33704 & 993 & 898 & 95 \\
\hline 19 & 34878 & 3657 & 31221 & 1474 & 1305 & 169 \\
\hline 20 & 33108 & 4926 & 28182 & 2331 & 2155 & 176 \\
\hline 21 & 31405 & 7823 & 23582 & 3033 & 2810 & 223 \\
\hline 22 & 32400 & 11993 & 20407 & 4296 & 4105 & 191 \\
\hline 23 & 33750 & 15452 & 18298 & 5340 & 5164 & 176 \\
\hline 24 & 31370 & 18467 & 12903 & 6286 & 6126 & 160 \\
\hline 25 & 32639 & 20805 & 11834 & 6784 & 6659 & 125 \\
\hline 26 & 33333 & 24037 & 9296 & 7329 & 7196 & 133 \\
\hline 27 & 37297 & 28530 & 8767 & 7360 & 7259 & 101 \\
\hline 28 & 33545 & 26452 & 7093 & 6249 & 6151 & 98 \\
\hline 29 & 31136 & 24991 & 6145 & 6153 & 6054 & 99 \\
\hline 30 & 34575 & 28664 & 5911 & 6151 & 6069 & 82 \\
\hline 31 & 33237 & 27610 & 5627 & 4693 & 4610 & 83 \\
\hline 32 & 31973 & 26929 & 5044 & 3794 & 3752 & 42 \\
\hline $\mathbf{3 3}$ & 28631 & 24199 & 4432 & 2832 & 2781 & 51 \\
\hline 34 & 25219 & 21445 & 3774 & 2564 & 2526 & 38 \\
\hline 35 & 33455 & 28514 & 4941 & 2713 & 2672 & 41 \\
\hline 36 & 21285 & 18039 & 3246 & 1604 & 1570 & 34 \\
\hline 37 & 25637 & 21876 & 3761 & 1662 & 1624 & 38 \\
\hline 38 & 27902 & 24043 & 3859 & 1536 & 1519 & 17 \\
\hline 39 & 31453 & 27053 & 4400 & 1326 & 1301 & 25 \\
\hline 40 & 29576 & 25491 & 4085 & 1214 & 1201 & 13 \\
\hline 41 & 29005 & 24649 & 4356 & 863 & 849 & 14 \\
\hline 42 & 29601 & 25126 & 4475 & 573 & 558 & 15 \\
\hline 43 & 29495 & 24637 & 4858 & 401 & 391 & 10 \\
\hline 44 & 28846 & 23966 & 4880 & 275 & 266 & 9 \\
\hline 45 & 30065 & 24626 & 5439 & 125 & 119 & 6 \\
\hline 46 & 28605 & 23087 & 5518 & 68 & 66 & 2 \\
\hline 47 & 29365 & 23792 & 5573 & 37 & 34 & 3 \\
\hline 48 & 26367 & 21058 & 5309 & 11 & 11 & 0 \\
\hline 49 & 26797 & 21361 & 5436 & 7 & 7 & 0 \\
\hline $15-49$ & 1087187 & 676405 & 410782 & 90891 & 88465 & 2426 \\
\hline$\%$ & 100 & $\quad 62,2$ & 37,8 & 100 & 97,3 & 2,7 \\
\hline
\end{tabular}

Fuente: Padrón de Habitantes y Movimiento Natural de Poblacion. 
FORMACIÓN DE LA PAREJA Y FECUNDIDAD: UNA SIMULACIÓN CON DATOS...

TABLA II

Mujeres y nacimientos de la Comunidad de Madrid en 1981

\begin{tabular}{|c|c|c|c|c|c|c|}
\hline \multirow{2}{*}{$\frac{1981}{\text { Edad }}$} & \multicolumn{3}{|c|}{ Mujeres } & \multicolumn{3}{|c|}{ Nacimientos } \\
\hline & Total & Casadas & No casadas & Total & $\begin{array}{c}\text { De madre } \\
\text { casada }\end{array}$ & $\begin{array}{l}\text { De madre } \\
\text { no casada }\end{array}$ \\
\hline 15 & 39746 & 226 & 39520 & 90 & 34 & 56 \\
\hline 16 & 40935 & 670 & 40265 & 252 & 150 & 102 \\
\hline 17 & 40372 & 1123 & 39249 & 543 & 378 & 165 \\
\hline 18 & 37736 & 1794 & 35942 & 880 & 667 & 213 \\
\hline 19 & 38284 & 3451 & 34833 & 1429 & 1180 & 249 \\
\hline 20 & 37950 & 5685 & 32265 & 2073 & 1808 & 265 \\
\hline 21 & 37940 & 9300 & 28640 & 2688 & 2453 & 235 \\
\hline 22 & 37306 & 12705 & 24601 & 3455 & 3220 & 235 \\
\hline 23 & 38146 & 17601 & 20545 & 4302 & 4070 & 232 \\
\hline 24 & 35389 & 19748 & 15641 & 4730 & 4523 & 207 \\
\hline 25 & 34849 & 22015 & 12834 & 5021 & 4822 & 199 \\
\hline 26 & 34926 & 24355 & 10571 & 5026 & 4870 & 156 \\
\hline 27 & 34486 & 24976 & 9510 & 5038 & 4876 & 162 \\
\hline 28 & 35551 & 26873 & 8678 & 4698 & 4553 & 145 \\
\hline 29 & 33931 & 26648 & 7283 & 4525 & 4388 & 137 \\
\hline 30 & 34659 & 27391 & 7268 & 4528 & 4355 & 173 \\
\hline 31 & 35825 & 28965 & 6860 & 3347 & 3239 & 108 \\
\hline 32 & 38733 & 31800 & 6933 & 3046 & 2956 & 90 \\
\hline 33 & 36928 & 30469 & 6459 & 2722 & 2629 & 93 \\
\hline 34 & 32506 & 27096 & 5410 & 2036 & 1975 & 61 \\
\hline 35 & 35525 & 29804 & 5721 & 1865 & 1799 & 66 \\
\hline 36 & 34166 & 28735 & 5431 & 1636 & 1569 & 67 \\
\hline 37 & 33992 & 28360 & 5632 & 1376 & 1328 & 48 \\
\hline 38 & 29482 & 24857 & 4625 & 1073 & 1033 & 40 \\
\hline 39 & 24921 & 20962 & 3959 & 697 & 673 & 24 \\
\hline 40 & 32083 & 27112 & 4971 & 590 & 568 & 22 \\
\hline 41 & 23574 & 19785 & 3789 & 456 & 436 & 20 \\
\hline 42 & 25153 & 21234 & 3919 & 254 & 241 & 13 \\
\hline 43 & 28067 & 23535 & 4532 & 179 & 171 & 8 \\
\hline 44 & 31110 & 26013 & 5097 & 125 & 124 & 1 \\
\hline 45 & 29667 & 24604 & 5063 & 71 & 68 & 3 \\
\hline 46 & 29559 & 24272 & 5287 & 35 & 34 & 1 \\
\hline 47 & 30627 & 24955 & 5672 & 20 & 19 & 1 \\
\hline 48 & 29967 & 24177 & 5790 & 9 & 9 & 0 \\
\hline 49 & 28727 & 23174 & 5553 & 5 & 5 & 0 \\
\hline $15-49$ & 1182818 & 714470 & 468348 & 68820 & 65223 & 3597 \\
\hline$\%$ & 100 & 60,4 & 39,6 & 100 & 94,8 & 5,2 \\
\hline
\end{tabular}

Fuente: Censo de Población y Movimiento Natural de Población. 
TABLA III

Mujeres y nacimientos de la Comunidad de Madrid en 1986

\begin{tabular}{|c|c|c|c|c|c|c|}
\hline \multirow{2}{*}{$\frac{1986}{\text { Edad }}$} & \multicolumn{3}{|c|}{ Mujeres } & \multicolumn{3}{|c|}{ Nacimientos } \\
\hline & Total & Casadas & No casadas & Total & $\begin{array}{c}\text { De madre } \\
\text { casada }\end{array}$ & $\begin{array}{l}\text { De madre } \\
\text { no casada }\end{array}$ \\
\hline 15 & 39980 & 197 & 39783 & 58 & 7 & 51 \\
\hline 16 & 40237 & 221 & 40016 & 157 & 50 & 107 \\
\hline 17 & 40116 & 474 & 39642 & 396 & 184 & 212 \\
\hline 18 & 40563 & 890 & 39673 & 735 & 424 & 311 \\
\hline 19 & 41197 & 1811 & 39386 & 1074 & 716 & 358 \\
\hline 20 & 39914 & 3003 & 36911 & 1329 & 1010 & 319 \\
\hline 21 & 40287 & 4900 & 35387 & 1595 & 1271 & 324 \\
\hline 22 & 39496 & 7094 & 32402 & 1964 & 1692 & 272 \\
\hline 23 & 38585 & 10560 & 28025 & 2523 & 2247 & 276 \\
\hline 24 & 38313 & 13838 & 24475 & 3146 & 2907 & 239 \\
\hline 25 & 38682 & 18212 & 20470 & 3754 & 3516 & 238 \\
\hline 26 & 37676 & 20944 & 16732 & 4302 & 4065 & 237 \\
\hline 27 & 37799 & 23629 & 14170 & 4540 & 4321 & 219 \\
\hline 28 & 38377 & 26063 & 12314 & 4405 & 4211 & 194 \\
\hline 29 & 35958 & 25967 & 9991 & 4235 & 4043 & 192 \\
\hline 30 & 35414 & 26444 & 8970 & 3882 & 3688 & 194 \\
\hline 31 & 34300 & 25884 & 8416 & 3095 & 2936 & 159 \\
\hline 32 & 34575 & 26950 & 7625 & 2562 & 2417 & 145 \\
\hline 33 & 35537 & 28121 & 7416 & 2212 & 2080 & 132 \\
\hline 34 & 33735 & 26876 & 6859 & 1819 & 1707 & 112 \\
\hline 35 & 33989 & 27568 & 6421 & 1581 & 1480 & 101 \\
\hline 36 & 34797 & 28222 & 6575 & 1274 & 1180 & 94 \\
\hline 37 & 36910 & 30419 & 6491 & 1145 & 1070 & 75 \\
\hline 38 & 36909 & 30620 & 6289 & 909 & 844 & 65 \\
\hline 39 & 31886 & 25977 & 5909 & 593 & 556 & 37 \\
\hline 40 & 35015 & 29103 & 5912 & 503 & 464 & 39 \\
\hline 41 & 34053 & 28126 & 5927 & 338 & 314 & 24 \\
\hline 42 & 33726 & 28057 & 5669 & 238 & 229 & 9 \\
\hline 43 & 29410 & 24580 & 4830 & 134 & 128 & 6 \\
\hline 44 & 24688 & 20353 & 4335 & 61 & 60 & 1 \\
\hline 45 & 29762 & 24566 & 5196 & 29 & 27 & 2 \\
\hline 46 & 25605 & 21263 & 4342 & 20 & 19 & 1 \\
\hline 47 & 24380 & 19995 & 4385 & 6 & 5 & 1 \\
\hline 48 & 26887 & 22381 & 4506 & 1 & 1 & 0 \\
\hline 49 & 30237 & 24696 & 5541 & 3 & 3 & 0 \\
\hline 15.49 & 1228995 & 678004 & 550991 & 54618 & 49872 & 4746 \\
\hline$\%$ & 100 & 55,2 & 44,8 & 100 & 91,3 & 8,7 \\
\hline
\end{tabular}

Fuente: Padrón de Habitantes y Movimiento Natural de Población. 
FORMACIÓN DE LA PAREJA Y FECUNDIDAD: UNA SIMULACIÓN CON DATOS...

TABLA IV

Mujeres y nacimientos de la Comunidad de Madrid en 1991

\begin{tabular}{|c|c|c|c|c|c|c|}
\hline \multirow{2}{*}{$\frac{1991}{\text { Edad }}$} & \multicolumn{3}{|c|}{ Mujeres } & \multicolumn{3}{|c|}{ Nacimientos } \\
\hline & Total & Casadas & No casadas & Total & $\begin{array}{c}\text { De madre } \\
\text { casada }\end{array}$ & $\begin{array}{l}\text { De madre } \\
\text { no casada }\end{array}$ \\
\hline 15 & 43405 & 62 & 43343 & 40 & 6 & 34 \\
\hline 16 & 43538 & 144 & 43394 & 125 & 24 & 101 \\
\hline 17 & 43292 & 240 & 43052 & 277 & 97 & 180 \\
\hline 18 & 43553 & 593 & 42960 & 423 & 201 & 222 \\
\hline 19 & 43214 & 1074 & 42140 & 633 & 360 & 273 \\
\hline 20 & 42408 & 2094 & 40314 & 797 & 483 & 314 \\
\hline 21 & 42069 & 3602 & 38467 & 1064 & 763 & 301 \\
\hline 22 & 41565 & 5246 & 36319 & 1253 & 991 & 262 \\
\hline 23 & 41987 & 8143 & 33844 & 1630 & 1370 & 260 \\
\hline 24 & 41781 & 11165 & 30616 & 2137 & 1844 & 293 \\
\hline 25 & 41830 & 14932 & 26898 & 2794 & 2511 & 283 \\
\hline 26 & 42099 & 18890 & 23209 & 3523 & 3194 & 329 \\
\hline 27 & 40992 & 21545 & 19447 & 4157 & 3830 & 327 \\
\hline 28 & 39851 & 23895 & 15956 & 4295 & 4010 & 285 \\
\hline 29 & 39362 & 25796 & 13566 & 4346 & 4050 & 296 \\
\hline 30 & 40070 & 27866 & 12204 & 4158 & 3877 & 281 \\
\hline 31 & 39239 & 28488 & 10751 & 3782 & 3526 & 256 \\
\hline 32 & 38854 & 28932 & 9922 & 3122 & 2886 & 236 \\
\hline 33 & 39167 & 29711 & 9456 & 2628 & 2419 & 209 \\
\hline 34 & 36598 & 28414 & 8184 & 2202 & 2022 & 180 \\
\hline 35 & 36257 & 28272 & 7985 & 1628 & 1460 & 168 \\
\hline 36 & 35110 & 27695 & 7415 & 1249 & 1104 & 145 \\
\hline 37 & 35129 & 27998 & 7131 & 947 & 834 & 113 \\
\hline 38 & 35636 & 28628 & 7008 & 729 & 647 & 82 \\
\hline 39 & 33660 & 27311 & 6349 & 494 & 433 & 61 \\
\hline 40 & 34852 & 28224 & 6628 & 364 & 308 & 56 \\
\hline 41 & 35476 & 28853 & 6623 & 259 & 227 & 32 \\
\hline 42 & 37664 & 30917 & 6747 & 170 & 151 & 19 \\
\hline 43 & 36248 & 29523 & 6725 & 114 & 97 & 17 \\
\hline 44 & 31878 & 26093 & 5785 & 42 & 36 & 6 \\
\hline 45 & 35472 & 29188 & 6284 & 20 & 16 & 4 \\
\hline 46 & 34089 & 27976 & 6113 & 13 & 10 & 3 \\
\hline 47 & 33269 & 27327 & 5942 & 2 & 1 & 1 \\
\hline 48 & 29373 & 24080 & 5293 & 2 & 1 & 1 \\
\hline 49 & 24462 & 19858 & 4604 & 0 & 0 & 0 \\
\hline $15-49$ & 1333449 & 692775 & 640674 & 49419 & 43789 & 5630 \\
\hline$\%$ & 100 & 52,0 & 48,0 & 100 & 88,6 & 11,4 \\
\hline
\end{tabular}

Fuente: Censo de Población y Movimiento Natural de Población. 
TABLA V

Mujeres y nacimientos de la Comunidad de Madrid en 1996

\begin{tabular}{|c|c|c|c|c|c|c|}
\hline \multirow{2}{*}{$\begin{array}{l}1996 \\
\text { Edad }\end{array}$} & \multicolumn{3}{|c|}{ Mujeres } & \multicolumn{3}{|c|}{ Nacimientos } \\
\hline & Total & Casadas & No casadas & Total & $\begin{array}{c}\text { De madre } \\
\text { casada }\end{array}$ & $\begin{array}{l}\text { De madre } \\
\text { no casada }\end{array}$ \\
\hline 15 & 34.378 & 180 & 34198 & 39 & 1 & 38 \\
\hline 16 & 36.892 & 237 & 36655 & 73 & 11 & 62 \\
\hline 17 & 39.066 & 307 & 38759 & 141 & 30 & 111 \\
\hline 18 & 41.517 & 421 & 41096 & 236 & 64 & 172 \\
\hline 19 & 43.780 & 623 & 43157 & 334 & 111 & 223 \\
\hline 20 & 44.760 & 1.055 & 43705 & 423 & 198 & 225 \\
\hline 21 & 44.618 & 1.626 & 42992 & 555 & 305 & 250 \\
\hline 22 & 44.291 & 2.614 & 41677 & 724 & 452 & 272 \\
\hline 23 & 43.903 & 4.085 & 39818 & 862 & 601 & 261 \\
\hline 24 & 44.363 & 6.502 & 37861 & 1185 & 890 & 295 \\
\hline 25 & 42.669 & 9.454 & 33215 & 1693 & 1373 & 320 \\
\hline 26 & 42.724 & 13.212 & 29512 & 2335 & 2044 & 291 \\
\hline 27 & 41.966 & 16.531 & 25435 & 2924 & 2627 & 297 \\
\hline 28 & 42.727 & 20.322 & 22405 & 3757 & 3406 & 351 \\
\hline 29 & 42.625 & 23.091 & 19534 & 4257 & 3930 & 327 \\
\hline 30 & 42.531 & 25.392 & 17139 & 4533 & 4206 & 327 \\
\hline 31 & 42.965 & 27.382 & 15583 & 4557 & 4218 & 339 \\
\hline 32 & 42.299 & 27.972 & 14327 & 4101 & 3794 & 307 \\
\hline 33 & 40.926 & 28.051 & 12875 & 3594 & 3310 & 284 \\
\hline 34 & 40.126 & 28.048 & 12078 & 2979 & 2718 & 261 \\
\hline 35 & 39.787 & 28.503 & 11284 & 2281 & 2066 & 215 \\
\hline 36 & 40.201 & 29.271 & 10930 & 1893 & 1692 & 201 \\
\hline 37 & 39.188 & 28.513 & 10675 & 1322 & 1152 & 170 \\
\hline 38 & 40.118 & 29.545 & 10573 & 966 & 832 & 134 \\
\hline 39 & 37.523 & 28.029 & 9494 & 701 & 581 & 120 \\
\hline 40 & 36.734 & 27.574 & 9160 & 452 & 380 & 72 \\
\hline 41 & 35.608 & 26.787 & 8821 & 268 & 226 & 42 \\
\hline 42 & 35.485 & 26.840 & 8645 & 145 & 118 & 27 \\
\hline 43 & 35.858 & 27.364 & 8494 & 83 & 67 & 16 \\
\hline 44 & 34.364 & 26.338 & 8026 & 35 & 29 & 6 \\
\hline 45 & 34.267 & 26.398 & 7869 & 20 & 13 & 7 \\
\hline 46 & 35.036 & 26.952 & 8084 & 7 & 7 & 0 \\
\hline 47 & 37.204 & 28.675 & 8529 & 3 & 1 & 2 \\
\hline 48 & 37.654 & 29.201 & 8453 & 4 & 3 & 1 \\
\hline 49 & 31.641 & 24.310 & 7331 & 1 & 1 & \\
\hline $15-49$ & 1424585 & 678307 & 746278 & 47483 & 41456 & 6027 \\
\hline$\%$ & 100 & 47,6 & 52,4 & 100 & 87,3 & 12,7 \\
\hline
\end{tabular}

Fuente: Padrón de Habitantes y Movimiento Natural de Población. 